\title{
OPTIMAL NATURAL DUALITIES: THE ROLE OF ENDOMORPHISMS
}

\author{
M. J. SARAMAGO \\ (Received 11 November 2002; revised 28 July 2003) \\ Communicated by B. A. Davey
}

\begin{abstract}
The optimality of dualities on a quasivariety $\mathscr{A}$, generated by a finite algebra $\underline{\mathbf{M}}$, has been introduced by Davey and Priestley in the 1990s. Since every optimal duality is determined by a transversal of a certain family of subsets of $\Omega$, where $\Omega$ is a given set of relations yielding a duality on $\mathscr{A}$, an understanding of the structures of these subsets-known as globally minimal failsets-was required. A complete description of globally minimal failsets which do not contain partial endomorphisms has recently been given by the author and $\mathrm{H}$. A. Priestley. Here we are concerned with globally minimal failsets containing endomorphisms. We aim to explain what seems to be a pattern in the way endomorphisms belong to these failsets. This paper also gives a complete description of globally minimal failsets whose minimal elements are automorphisms, when $\underline{\mathbf{M}}$ is a subdirectly irreducible lattice-structured algebra.
\end{abstract}

2000 Mathematics subject classification: primary 06D05, 06D30, 08C15.

Keywords and phrases: natural duality, optimal duality, failset.

\section{Introduction}

A general theory of natural dualities started to emerge within universal algebra in the mid 1970s and since then it has been rapidly developed. The primary aim of this theory is to obtain a representation of the algebras in a quasivariety $\mathscr{A}$ generated by an algebra $\underline{\mathbf{M}}$, as algebras of continuous structure-preserving maps into a convenient structure $\underset{\sim}{\mathbf{M}}$ on the underlying set $M$ of $\underline{\mathbf{M}}$. A natural duality for $\mathscr{A}$ gives us a uniform method to get such a representation for each one of the elements of $\mathscr{A}$, in which case we say that the structure $\underline{\mathbf{M}}$ dualises $\underline{\mathbf{M}}$. (We refer to [1] for developments and basic facts of the theory.) The optimality of dualities on a quasivariety $\mathscr{A}=\mathbb{U S P}(\underline{\mathbf{M}})$,

The author is a member of the project POCTI/32440/MAT/2000 and of CAUL.

(C) 2004 Australian Mathematical Society 1446-7887/04 \$A2.00+0.00 
where $\underline{\mathbf{M}}$ is a finite algebra, has firstly been investigated by Davey and Priestley (see $[3,4])$. Relative to a given set $\Omega$ of relations yielding a duality, they characterized the optimal dualities as the dualities determined by the transversals of a certain family of subsets of $\Omega$. However the structure of these subsets-known as globally minimal failsets - remained to be understood. The development of the theory presented in [4] progressed, symbiotically, alongside computer calculations of the globally minimal failsets relative to $\mathbb{S}\left(\underline{\mathbf{M}}^{2}\right)$ for particular distributive-lattice-ordered algebras $\underline{\mathbf{M}}$. In spite of the limitations on the size of examples that can be handled, the computergenerated results suggest some common characteristics of these failsets. Somehow this has showed us which direction to take in order to get a structural description of globally minimal failsets in the case that $\$\left(\underline{\mathbf{M}}^{2}\right)$ dualises $\underline{\mathbf{M}}$, as it does whenever $\underline{\mathbf{M}}$ has a definable lattice structure. To concentrate on analysing the globally minimal failsets that do not contain any partial endomorphism of $\underline{\mathbf{M}}$-called pe-free globally minimal failsets - seemed to be a natural first step. In [9] a complete description of pe-free globally minimal failsets is given, for the special cases where condition $(\mathrm{H})$ holds. This condition states that the elements of the duals $D(\mathbf{r})$, with $\mathbf{r} \in \mathbb{S}\left(\underline{\mathbf{M}}^{2}\right)$, are composites of the restrictions $\rho_{i}$ to $r$ of the projection maps with partial endomorphisms of $\underline{\mathbf{M}}$. Following the work presented in [9], now we focus on globally minimal failsets that contain partial endomorphisms. Among the examples we know, there are globally minimal failsets whose minimal elements are exclusively endomorphisms and the converses of their graphs. This makes us think that we should start to understand first the structure of these particular ones. In certain cases the endomorphisms are automorphisms living outside the same maximal subgroup of the group Aut $\underline{\mathbf{M}}$ of automorphisms of $\underline{\mathbf{M}}$. The structure of these failsets is described here in Section 3 . In case $\underline{M}$ is a subdirectly irreducible lattice-structured algebra, as it happens to be in many of the examples we know, we prove that the set of minimal elements of any globally minimal failset, whose minimal elements are automorphisms, is the complement of a maximal proper subgroup of Aut $\underline{\mathbf{M}}$; conversely, the complement of each maximal proper subgroup of Aut $\underline{\mathbf{M}}$ is the set of minimal elements of one such globally minimal failset. In Section $\mathbf{4}$, under the assumption that $\underline{\mathbf{M}}$ is a subdirectly irreducible lattice-structured algebra, we prove that these globally minimal failsets are exactly those that contain automorphisms among their minimal elements. So we concentrate on aut-free globally minimal failsets, that is, globally minimal failsets that do not intersect Aut $\underline{\mathbf{M}}$ but whose minimal elements are endomorphisms and converses of their graphs. As expected, their structure is not so easy to describe as the structure of globally minimal failsets described in [9]; it depends on the covers $[a, b]_{x}$ of the intersection of the kernels of endomorphisms of $\underline{\mathbf{M}}$ outside Aut $\underline{\mathbf{M}}$. We show that the aut-free globally minimal failsets are unions of sets, each of them depending on one of the endomorphisms that separate $a$ and $b$ and belong to a given generating set of the monoid End $\underline{\mathbf{M}}$ of endomorphisms of $\underline{\mathbf{M}}$. 


\section{Preliminaries}

Throughout the paper we assume that $\underline{\mathbf{M}}$ is a given finite algebra of type $F$, and we take $\mathscr{A}=\mathbb{B S P}(\underline{\mathbf{M}})$ to be the quasivariety generated by $\underline{\mathbf{M}}$; in the examples we refer to, $\mathscr{A}=\mathbb{U S P}(\underline{\mathbf{M}})$ is often a variety.

An $n$-ary relation $s$ on $M$ is called algebraic if $s$ is a subalgebra of the direct power $\underline{\mathrm{M}}^{n}$; we write $\mathbf{s}$ when we want to think of $s$ as a member of $\mathscr{A}$. For a given set $R$ of algebraic relations on $\underline{\mathbf{M}}$, define $\underset{\sim}{\mathbf{M}}:=(M ; R, \tau)$ to be the topological relational structure on the underlying set $M$ of $\underline{\mathbf{M}}$ in which $\tau$ is the discrete topology. Let $\mathscr{X}=\| S_{c} \mathbb{P}(\underset{\sim}{\mathbf{M}})$ be the category in which objects are all isomorphic copies of closed substructures of powers of $\mathbf{M}$ and in which morphisms are the continuous $R$-preserving maps. Then we consider the hom functors $D=\mathscr{A}(-, \underline{\mathbf{M}}): \mathscr{A} \rightarrow \mathscr{X}$ and $E=\mathscr{X}(-, \mathbf{M}): \mathscr{X} \rightarrow \mathscr{A}$. The dual space $D(\mathbf{A})$ of each $\mathbf{A} \in \mathscr{A}$ belongs to $\mathscr{X}=\mathbb{I} \mathbb{S}_{c} \mathbb{P}(\mathbf{M})$, and therefore its topology is induced by the topology of $\underset{\sim}{\mathbf{M}}$ and its structure is given by the set of the pointwise-defined relations on $D(\mathbf{A})$ : if $r \in R$ is an $n$-ary relation, then $\left(x_{1}, \ldots, x_{n}\right) \in r$ on $D(A)$ if and only if $\left(x_{1}(a), \ldots, x_{n}(a)\right) \in r$, for all $a \in A$. We say that $\underset{\mathcal{M}}{\mathbf{M}}$ (or, alternatively, $R$ ) yields a (natural) duality on $\underline{\mathbf{M}}$ (or dualises $\underline{\mathbf{M}}$ ) if every $\mathbf{A} \in \mathscr{A}$ is isomorphic to its second dual $E D(\mathbf{A})$. This setting is less general than that treated in [5], where operations (total or partial) are included in the dualising structure as well relations. Here we encompass $n$-ary operations by including in $R$ their graphs (which are subalgebras of $\underline{\mathbf{M}}^{n+1}$ ); this is admissible because we consider dualities, rather than strong dualities (see [1, pages 40-41, 63]). It allows us, in particular, to treat as interchangeable any partial endomorphism $h$ of $\underline{\mathbf{M}}$ and the associated binary relation graph $h$.

In this work we are concerned only with cases where a duality is known to exist. This occurs in particular if $\underline{\mathbf{M}}$ has a $(k+1)$-ary near-unanimity term, with $k \geqslant 2$, and then $\mathbf{M}=\left(M ; \mathbb{S}\left(\underline{\mathbf{M}}^{k}\right), \tau\right)$ yields a duality on $\mathscr{A}$. This very useful result, the NU-Duality Theorem, is due to Davey and Werner (see [1,5]); it applies in particular whenever $\underline{\mathbf{M}}$ has an underlying lattice structure and in that case asserts that $\mathbb{S}\left(\underline{\mathbf{M}}^{2}\right)$ dualises $\mathbf{M}$. The NU-Duality Theorem is a valuable existence theorem for dualities. However, even when $k=2$ and $M$ is small, it supplies dualities which, except in the simplest cases, contain extremely large numbers of relations, and such dualities are obviously of little practical use. It is therefore natural to ask how a duality based on some given dualising set $\Omega$ might be simplified by deleting superfluous relations. In fact, we would like to know how to get a subset $R$ of $\Omega$ that yields an optimal duality on $\underline{\mathbf{M}}$, in the sense that $R$ yields a duality on $\underline{\mathbf{M}}$ but no proper subset of $R$ does so. The characterization of all the subsets of a given dualising set which yield optimal dualities on $\underline{\mathbf{M}}$ is one of central problems in the natural duality theory. The solution of this problem relies on the analysis of entailment: a subset $R$ of $\bigcup_{n \geq 1} \mathbb{S}\left(\mathbf{M}^{n}\right)$ entails a relation $r$ (in symbols, $R \vdash r$ ) if, for every $\mathbf{A} \in \mathscr{A}$, every continuous map 
$\varphi: D(\mathbf{A}) \rightarrow M$ which preserves every relation in $R$ also preserves $r$. Locally, for a fixed $\mathrm{s} \in \mathscr{A}$, we say that $R$ entails $r$ (on $D(\mathrm{~s})$ ) if every continuous $R$-preserving map $\varphi: D(\mathbf{s}) \rightarrow M$ also preserves $r$. If $R$ dualises $\underline{\mathbf{M}}$ and $r \in R$ is entailed by $R \backslash\{r\}$, then we may delete $r$ from $R$ and we still have a duality on $\underline{\mathbf{M}}$, given by $R \backslash\{r\}$. Hence it was important for the relations entailed by $R$ to be described intrinsically. This was achieved by Davey, Haviar and Priestley in [2]. A key tool in this work is the Test Algebra Lemma, which shows that entailment is a local matter.

LeMma 2.1 (Test Algebra Lemma, [4, 2.4]). Let $\Omega=\bigcup_{n \geqslant 1} \$\left(\mathbf{M}^{n}\right)$. For every $R \subseteq \Omega$ and $s \in \Omega$, we have that $R$ entails $s$ if and only if $R$ entails $s$ on $D(\mathbf{s})$.

Let $\Omega \subseteq \bigcup_{n \geq 1} \subseteq\left(\mathbf{M}^{n}\right)$ be a fixed set of algebraic relations on $\underline{\mathbf{M}}$. For every $r \in \Omega$ and every map $u: D(\mathbf{r}) \rightarrow M$, define

$$
U=\operatorname{Fail}_{\mathrm{r}}(u):=\{s \in \Omega \mid u \text { fails to preserve } s\} ;
$$

the set $U$ is called a failset of $r$ (within $\Omega$ ) if it contains $r$ and it is called a failset whenever it is a failset of some $s \in U$.

Let $R \subseteq \Omega$ and $r \in \Omega$. Observe that if $R$ entails $r$ and $U$ is a failset of $r$ then $U$ is a failset of some $s \in R$. The map $R \mapsto \bar{R}:=\{r \in \Omega \mid R \vdash r\}$ is a closure operator, referred to as entailment closure. From the definition, it is immediate that the complement of Fail $_{\mathbf{r}}(u)$ is closed in $\Omega$, for every map $u: D(\mathbf{r}) \rightarrow M[4,3.1]$. There are various ways of building relations which are entailed by a given subset $R$ of $\Omega$. Next we list the constructs we need for this work. They are particular cases of some of the available constructs presented in [2], with $n=2$.

Trivial relations. Construct two trivial relations $\Delta_{M}:=\{(c, c) \mid c \in M\}$ and $M^{2}$.

Converse. From a binary relation $r$ construct $r^{-}=\left\{(c, d) \in M^{2} \mid(d, c) \in r\right\}$.

Intersection. From binary relations $r$ and $s$, construct $r \cap s$.

Product. From $N_{1}, N_{2} \subseteq M$ construct $N_{1} \times N_{2}$.

Domains. From a partial endomorphism $e: \mathbf{N} \rightarrow \mathbf{M}$, construct the domain, dom $e$, of $e$.

Action by partial endomorphisms. From $r \subseteq \mathbf{M}^{2}$ and a partial endomorphism $e: \mathbf{N} \rightarrow \mathbf{M}$, with $\mathbf{N} \leq \mathbf{M}$, construct

$$
e \cdot r:=\left\{\left(c_{1}, c_{2}\right) \in M^{2} \mid c_{1} \in N \text { and }\left(e\left(c_{1}\right), c_{2}\right) \in r\right\} .
$$

If $r$ is the graph of an endomorphism, then $e \cdot r$ is the composition of $e$ and $r$ qua maps, with $e$ done first, that is $r \circ e$. In case $r$ is unary we define the action of $e$ on $r$ to be the natural analogue of this, namely

$$
e^{-1}(r):=\{c \in \mathbf{N} \mid e(c) \in r\} .
$$


In case $r$ is binary and $e, f$ are partial endomorphisms, note that

$$
(e \times f)^{-1}(r):=\{(c, d) \in \operatorname{dom} e \times \operatorname{dom} f \mid(e(c), f(d)) \in r\}
$$

is $\left(f \cdot\left((e \cdot r)^{\smile}\right)\right)^{\smile}$ and so it is an available construct. In particular, if $e, f \in$ Aut $\underline{\mathbf{M}}$ then $(e \times f)(r)=\left(e^{-1} \times f^{-1}\right)^{-1}(r)$ is an available construct. We often denote by $\operatorname{ker}(e, f)$ the set $(e \times f)^{-1}\left(\Delta_{M}\right)$ and by $e(r)$ the set $(e \times e)(r)$, where $e \in$ Aut $\underline{\mathbf{M}}$.

For a given dualising set $\Omega$ of algebraic relations on $\underline{\mathbf{M}}$, let $R \subseteq \Omega$ yield an optimal duality on $\underline{\mathbf{M}}$. For every $r \in R$, we have that $R \backslash\{r\}$ does not entail $r$, that is, by the Test Algebra Lemma, $R \backslash\{r\}$ does not entail $r$ on $D(\mathbf{r})$, and so there exists a map $u: D(\mathbf{r}) \rightarrow M$ such that the set Fail $_{\mathbf{r}}(u)$ contains $r$ but does not intersect $R \backslash\{r\}$. Hence, for every $r \in R$, there exists a minimal failset Fail $_{\mathbf{r}}(u)$ of $r$ such that $r$ is the unique element of $R$ in $\operatorname{Fail}_{\mathrm{r}}(u)$. Let $U \subseteq \Omega$ be a failset. We say that $U$ is a minimal failset of $r$ if $U$ is a minimal element of the set of all failsets of $r$. If $U$ is also a minimal element of the set of all failsets, then $U$ is called a globally minimal failset. (Subsets of $\Omega$ are always ordered by inclusion.)

The following results are Corollary 3.6 and part of Theorem 3.14 of [4].

PROPOSITION 2.2. Let $s \in \Omega$. Let $U=\operatorname{Fail}_{\mathbf{r}}(u)$ be a failset containing $s$. Then there is a minimal failset $U_{s}$ of $s$ with $U_{s} \subseteq U$.

THEOREM 2.3. Let $\emptyset \neq U \subseteq \Omega$. Then the following are equivalent:

(a) $U$ is a globally minimal failset;

(b) $U$ is a minimal failset of $r$ for all $r \in U$.

In [4], Davey and Priestley demonstrated how optimal dualities are obtained from the globally minimal failsets. Let $\mathscr{G}$ be the family of all globally minimal failsets within $\Omega$ and let $T$ be a subset of $\Omega$. The set $T$ is called a transversal of $\mathscr{G}$ if $T$ intersects each $U \in \mathscr{G}$ but no proper subset of $T$ does. The next theorem is part of Theorem 4.4 (the Optimal Duality Theorem) of [4].

THEOREM 2.4. Assume that $\Omega$ is finite and yields a duality on $\mathbb{B P}(\underline{\mathbf{M}})$. Then the following are equivalent:

(a) $R \subseteq \Omega$ yields an optimal duality on $\underline{\mathbf{M}}$;

(b) $R$ is a transversal of the globally minimal failsets in $\Omega$.

Thus, when $\Omega$ is finite and yields a duality on $\underline{\mathbf{M}}$, in order to obtain the optimal dualities on $\mathscr{A}$ we start by determining the globally minimal failsets and then take the transversals of $\mathscr{G}$. For further details see [4] and [1].

Onwards we assume that $\Omega=\mathbb{S}\left(\underline{\mathbf{M}}^{2}\right)$ yields a duality on $\underline{\mathbf{M}}$. Given a failset $U$, we denote by $U_{\min }$ the set of minimal elements of $U$. 


\section{Globally minimal failsets with automorphisms as minimal elements}

In the examples of globally minimal failsets within $\mathbb{S}\left(\underline{\mathbf{M}}^{2}\right)$ that we already know, we almost always find globally minimal failsets for which the set of minimal elements is the complement of a maximal proper subgroup of Aut $\underline{\mathbf{M}}$. That is the case of $\underline{\mathbf{M}}=\mathbf{P}_{m, n}$, the distributive double $\mathrm{p}$-algebra given by the ordinal sum of the $\mathrm{m}$-atom Boolean lattice and the n-atom Boolean lattice, presented in [7], or of $\underline{\mathbf{M}}$ being the four-element generating algebra of the variety of de Morgan algebras, presented in [9, Section 6], or even of the non-distributive cases presented in [10, Chapter 2], such as the diamond $\mathbf{M}_{3}$ and the modular ortholattice $\mathbf{M O}_{4}$. In this section we present some general results that explain these particular cases.

The first result we present gives us a necessary condition on a map $u:$ End $\underline{\mathbf{M}} \rightarrow M$ for Fail ${ }_{g}(u)$ to be a failset of $g \in$ Aut $\underline{\mathbf{M}}$ whose minimal elements are automorphisms. Observe that the relations $r$ in such a failset must contain the graph of some automorphism and $r \in$ Fail $_{g}(u)$ must be witnessed by a pair of automorphisms of $\underline{\mathbf{M}}$. So they are neither graphs of proper partial endomorphisms of $\underline{\mathbf{M}}$ nor graphs of endomorphisms in End $\underline{\mathbf{M}} \backslash$ Aut $\underline{\mathbf{M}}$.

In case End $\underline{\mathbf{M}} \neq$ Aut $\underline{\mathbf{M}}$, we denote by $\kappa$ the following congruence relation on $\underline{\mathbf{M}}$

$$
\bigcap(\operatorname{ker} f \mid f \in \text { End } \underline{\mathbf{M}} \backslash \text { Aut } \underline{\mathbf{M}}) \text {. }
$$

PROPOSITION 3.1. Let $u:$ End $\underline{\mathbf{M}} \rightarrow M$ be a map such that Fail $_{g}(u)$ is a failset of $g \in$ Aut $\underline{\mathbf{M}}$ whose minimal elements are automorphisms of $\underline{\underline{\mathbf{M}}}$. If End $\underline{\mathbf{M}} \neq$ Aut $\underline{\mathbf{M}}$ then, for every $x, y \in$ Aut $\underline{\mathbf{M}}\left(u(x), y^{-1}(u(y \circ x)) \in \kappa ;\right.$ consequently $\kappa \neq \Delta_{M}$.

Proof. Take $x, y \in$ Aut $\underline{\mathbf{M}}$ and let $f \in$ End $\underline{\mathbf{M}} \backslash$ Aut $\underline{\mathbf{M}}$. Since $f, f \circ y^{-1} \notin$ Fail $_{g}(u)$, we have that $f(u(x))=u(f \circ x)=u\left(f \circ y^{-1} \circ y \circ x\right)=\left(f \circ y^{-1}\right)(u(y \circ x))$, and so $\left(u(x), y^{-1}(u(y \circ x)) \in \operatorname{ker} f\right.$. In particular, for $y=g$ and $x \in$ Aut $\underline{\mathrm{M}}$ such that $(x, g \circ x)$ witnesses $g \in$ Fail $_{g}(u)$, we have that $\left(u(x), g^{-1}(u(g \circ x))\right) \in \kappa \backslash \triangle_{M}$.

Now take $U$ to be a globally minimal failset and suppose that the set $U_{\min }$ of minimal elements of $U$ is a set of automorphisms of $\underline{\mathbf{M}}$.

Let $[a, b]$ denote the subalgebra of $\underline{\mathbf{M}}^{2}$ generated by $\Delta_{M} \cup\{(a, b)\}$, where $(a, b) \in$ $M^{2}$. Recall that, given $x \in$ Aut $\underline{\mathbf{M}}$, we denote $(x \times x)([a, b])$ by $x([a, b])$.

PROPOSITION 3.2. There exists a map $u:$ End $\underline{M} \rightarrow M$ such that $U=$ Fail $_{g}(u)$, for some $g \in U$, and for every $x, y \in$ Aut $\underline{\mathbf{M}}$

$$
u(y) \neq\left(y \circ x^{-1}\right)(u(x)) \Longrightarrow\left[x^{-1}(u(x)), y^{-1}(u(y))\right] \succ \Delta_{M} .
$$

Proof. Take $g \in U_{\min }$ and take $u:$ End $\underline{\mathbf{M}} \rightarrow M$ to be a map such that $U=$ Fail $_{g}(u)$. Let $x, y \in$ Aut $\underline{\mathbf{M}}$ satisfy $(u(x), u(y)) \notin g^{\prime}$, where $g^{\prime}:=y \circ x^{-1}$. Take 
$a^{\prime}=x^{-1}(u(x))$ and $b^{\prime}=y^{-1}(u(y))$. Note that $\left[a^{\prime}, b^{\prime}\right]>\Delta_{M}$ because $y\left(a^{\prime}\right) \neq y\left(b^{\prime}\right)$. Suppose that $\left[a^{\prime}, b^{\prime}\right]$ does not cover $\Delta_{M}$. There exists $\left(a^{*}, b^{*}\right) \in M^{2}$ such that $\left[a^{\prime}, b^{\prime}\right]>\left[a^{*}, b^{*}\right]>\Delta_{M}$. Since $\left(a^{*}, b^{*}\right) \in\left[a^{\prime}, b^{\prime}\right]$ there exist an m-ary term $t$ of type $F$ and $\left(a_{1}, b_{1}\right), \ldots\left(a_{m}, b_{m}\right) \in M^{2}$, with either $a_{i}=b_{i}$ or $\left(a_{i}, b_{i}\right)=\left(a^{\prime}, b^{\prime}\right)$, for $i \in(1, \ldots, m)$, such that

$$
\left(a^{\star}, b^{*}\right)=t^{M^{2}}\left(\left(a_{1}, b_{1}\right), \ldots,\left(a_{m}, b_{m}\right)\right)=\left(t^{M}\left(a_{1}, \ldots, a_{m}\right), t^{M}\left(b_{1}, \ldots, b_{m}\right)\right) .
$$

Define a map $\alpha: M \rightarrow M$ by $\alpha(c)=t^{M}\left(c_{1}, \ldots, c_{m}\right)$, where

$$
c_{i}= \begin{cases}a_{i} & \text { if } a_{i}=b_{i} \\ c & \text { otherwise }\end{cases}
$$

Now define a map $v:$ End $\underline{\mathbf{M}} \rightarrow M$ as follows

$$
v(z)= \begin{cases}z\left(\alpha\left(a^{\prime}\right)\right) & \text { if } z \notin \text { Aut } \underline{\mathbf{M}} ; \\ z(\alpha(c)) & \text { otherwise, where } c=z^{-1}(u(z)) .\end{cases}
$$

Note that $(v(x), v(y))=\left(x\left(\alpha\left(a^{\prime}\right)\right), y\left(\alpha\left(b^{\prime}\right)\right)\right)=\left(x\left(a^{\star}\right), y\left(b^{\star}\right)\right) \notin g^{\prime}$. Hence Fail $g_{g^{\prime}}(v)$ is a failset of $g^{\prime}$. Observe that for every $z \in$ Aut $\underline{\mathbf{M}}$ and every $f \in$ End $\underline{\mathbf{M}} \backslash$ Aut $\underline{\mathbf{M}}$, we have that $f \circ z \circ x^{-1}, f \notin U$ and then

$$
u(f \circ z)=\left(f \circ z \circ x^{-1}\right)(u(x))=(f \circ z)\left(a^{\prime}\right)
$$

and $u(f \circ z)=f(u(z))$. Hence $\left(c, a^{\prime}\right) \in \operatorname{ker}(f \circ z)$, where $c=z^{-1}(u(z))$. So $\left(\alpha(c), \alpha\left(a^{\prime}\right)\right) \in \operatorname{ker}(f \circ z)$ and consequently

$$
v(f \circ z)=(f \circ z)\left(\alpha\left(a^{\prime}\right)\right)=(f \circ z)(\alpha(c))=f(v(z)) .
$$

Thus Fail $g_{g^{\prime}}(v) \cap E n d \underline{\mathbf{M}} \subseteq$ Aut $\underline{\mathbf{M}}$. Now let $s \in \operatorname{Fail}_{g^{\prime}}(v)$. We want to prove that $s \in U$. There exist $x^{\prime}, y^{\prime} \in$ Aut $\underline{\mathbf{M}}$ such that $\left(x^{\prime}, y^{\prime}\right) \in s$ and $\left(v\left(x^{\prime}\right), v\left(y^{\prime}\right)\right) \notin s$. Observe that

$$
\begin{aligned}
\left(v\left(x^{\prime}\right), v\left(y^{\prime}\right)\right) & =\left(x^{\prime}(\alpha(c)), y^{\prime}(\alpha(d))\right) \\
& =\left(x^{\prime}\left(t^{M}\left(c_{1}, \ldots, c_{m}\right)\right), y^{\prime}\left(t^{M}\left(d_{1}, \ldots, d_{m}\right)\right)\right) \\
& =\left(t^{M}\left(x^{\prime}\left(c_{1}\right), \ldots, x^{\prime}\left(c_{m}\right)\right), t^{M}\left(y^{\prime}\left(d_{1}\right), \ldots, y^{\prime}\left(d_{m}\right)\right)\right) \\
& =t^{M^{2}}\left(\left(x^{\prime}\left(c_{1}\right), y^{\prime}\left(d_{1}\right)\right), \ldots,\left(x^{\prime}\left(c_{m}\right), y^{\prime}\left(d_{m}\right)\right)\right)
\end{aligned}
$$

with $x^{\prime}(c)=u\left(x^{\prime}\right)$ and $y^{\prime}(d)=u\left(y^{\prime}\right)$ and either $c_{i}=d_{i}=a_{i}=b_{i}$ or $\left(c_{i}, d_{i}\right)=$ $(c, d)$. Since $\left(v\left(x^{\prime}\right), v\left(y^{\prime}\right)\right) \notin s$, we must have $\left(x^{\prime}\left(c_{i}\right), y^{\prime}\left(d_{i}\right)\right) \notin s$, for some $i \in(1, \ldots, m)$. Note also that $\left(x^{\prime}, y^{\prime}\right) \in s$ implies that $\left(x^{\prime}\left(c_{i}\right), y^{\prime}\left(d_{i}\right)\right) \in s$ whenever $c_{i}=d_{i}$. Thus we have $\left(x^{\prime}(c), y^{\prime}(d)\right) \notin s$, that is, $\left(u\left(x^{\prime}\right), u\left(y^{\prime}\right)\right) \notin s$. Now, by the 
minimality of $U$ as a failset of $g^{\prime}$, we have that $U=$ Fail $_{g^{\prime}}(v)$. Moreover, for every $z, w \in$ Aut $\underline{\mathbf{M}}$, we have that

$$
\begin{aligned}
\left(z^{-1}(v(z)), w^{-1}(v(w))\right) & =\left(\alpha\left(z^{-1}(u(z))\right), \alpha\left(w^{-1}(u(w))\right)\right) \\
& \in\left[z^{-1}(u(z)), w^{-1}(u(w))\right]
\end{aligned}
$$

and consequently, if $\left[z^{-1}(u(z)), w^{-1}(u(w))\right] \succ \Delta_{M}$ then either

$$
\left[z^{-1}(v(z)), w^{-1}(v(w))\right]=\Delta_{M} \quad \text { or } \quad\left[z^{-1}(v(z)), w^{-1}(v(w))\right] \succ_{M}
$$

We may go on constructing maps $v$ until we get $\left[x^{-1}(v(x)), y^{-1}(v(y))\right] \succ \Delta_{M}$ for every $x, y \in$ Aut $\underline{\mathbf{M}}$ such that $v(y) \neq\left(y \circ x^{-1}\right)(v(x))$.

COROLlary 3.3. There exist a map $u:$ End $\underline{\mathbf{M}} \rightarrow M$ and $g \in U$ such that $U=$ Fail $_{g}(u), u(g) \neq g\left(u\left(\operatorname{id}_{M}\right)\right)$ and for every $x, y \in$ Aut $\underline{\mathbf{M}}$

$$
u(y) \neq\left(y \circ x^{-1}\right)(u(x)) \Longrightarrow\left[x^{-1}(u(x)), y^{-1}(u(y))\right] \succ \Delta_{M}
$$

ProOf. By the previous proposition, we may take a map $u:$ End $\underline{\mathbf{M}} \rightarrow M$ and $g \in U$ to satisfy $U=\operatorname{Fail}_{g}(u)$ and for every $x, y \in$ Aut $\underline{\mathbf{M}}$

$$
u(y) \neq\left(y \circ x^{-1}\right)(u(x)) \Longrightarrow\left[x^{-1}(u(x)), y^{-1}(u(y))\right] \succ \Delta_{M}
$$

If $u(g)=g\left(u\left(\mathrm{id}_{M}\right)\right.$ then we define a map $v:$ End $\underline{\mathbf{M}} \rightarrow M$ by $v(x)=u(x \circ z)$, where $z$ is a fixed automorphism of $\underline{\mathbf{M}}$ satisfying $u(g \circ z) \neq g(u(z))$. Then $v(g) \neq g\left(v\left(\mathrm{id}_{M}\right)\right)$ and $U=$ Fail $_{g}(v)$. Moreover, we still have that for every $x, y \in$ Aut $\underline{\mathbf{M}}$

$$
v(y) \neq\left(y \circ x^{-1}\right)(v(x)) \Longrightarrow\left[x^{-1}(v(x)), y^{-1}(v(y))\right] \succ \Delta_{M}
$$

because $z([a, b]) \succ \Delta_{M}$ whenever $[a, b] \succ \Delta_{M}$.

Let $\boldsymbol{u}:$ End $\underline{\mathbf{M}} \rightarrow M$ be a map and let $g \in U_{\min }$ such that the conditions of Corollary 3.3 hold.

If the algebra $\underline{\mathbf{M}}$ has a definable lattice structure then the covers of $\Delta_{M}$ in $\mathbb{S}\left(\underline{\mathbf{M}}^{2}\right)$, and in particular the covers $\left[x^{-1}(u(x)), y^{-1}(u(y))\right]$, are of the form $\left[c, c^{-}\right]$or $\left[c^{-}, c\right]$, for $c \in \mathscr{J}(\underline{\mathbf{M}})$, where $\mathscr{J}(\underline{\mathbf{M}})$ is the set of join-irreducible elements of $(M ; \vee, \wedge)$ and $c^{-}$denotes the unique element in $M$ covered by $c$ (see [6], Proposition 1.6). Next we see how this lemma allows us to restrict the possible choices of $u$.

Suppose that $\underline{M}$ is a lattice-structured algebra. By Lemma 1.5 of [6], there exists $c \in \mathscr{J}(\underline{\mathbf{M}})$ such that $\left(\left(u\left(\operatorname{id}_{M}\right), g^{-1}(u(g))\right) \wedge(c, c)\right) \vee\left(c^{-}, c^{-}\right) \in\left\{\left(c, c^{-}\right),\left(c^{-}, c\right)\right\}$ and so $\left[u\left(\operatorname{id}_{M}\right), g^{-1}(u(g))\right] \in\left\{\left[c, c^{-}\right],\left[c^{-}, c\right]\right\}$. 
Define a map $u^{\prime}$ : End $\underline{\mathbf{M}} \rightarrow M$ by $u^{\prime}(x)=(u(x) \wedge x(c)) \vee x\left(c^{-}\right)$. We claim that $U=$ Fail $_{g}\left(u^{\prime}\right)$. Note that

$$
\begin{aligned}
\left(u^{\prime}\left(\operatorname{id}_{M}\right), u^{\prime}(g)\right) & =\left(\left(u\left(\operatorname{id}_{M}\right) \wedge c\right) \vee c^{-},(u(g) \wedge g(c)) \vee g\left(c^{-}\right)\right) \\
& =\left(\left(u\left(\operatorname{id}_{M}\right) \wedge c\right) \vee c^{-}, g\left(\left(g^{-1}(u(g)) \wedge c\right) \vee c^{-}\right)\right) \\
& \in\left\{\left(c, g\left(c^{-}\right)\right),\left(c^{-}, g(c)\right)\right\}
\end{aligned}
$$

and hence $u^{\prime}(g) \neq g\left(u^{\prime}\left(\operatorname{id}_{M}\right)\right)$. Then Fail ${ }_{g}\left(u^{\prime}\right)$ is a failset of $g$. Take $s \in$ Fail $_{g}\left(u^{\prime}\right)$. There exist $x, y \in$ End $\underline{\mathbf{M}}$ such that $(x, y) \in s$ and $\left(u^{\prime}(x), u^{\prime}(y)\right) \notin s$. Since

$$
\left(u^{\prime}(x), u^{\prime}(y)\right)=((u(x), u(y)) \wedge(x(c), y(c))) \vee\left(x\left(c^{-}\right), y\left(c^{-}\right)\right)
$$

and $(x(c), y(c)),\left(x\left(c^{-}\right), y\left(c^{-}\right)\right) \in s$, we must have that $(u(x), u(y)) \notin s$ and so $s \in U$. Thus Fail $g_{g}\left(u^{\prime}\right) \subseteq U$ and, by the minimality of $U$, we get $U=$ Fail $_{g}\left(u^{\prime}\right)$.

Let $x \in$ Aut $\underline{\text { M. Then either }}$

$$
\begin{aligned}
& x^{-1}(u(x)) \wedge c=c \quad \text { and so } \quad u^{\prime}(x)=x\left(\left(x^{-1}(u(x)) \wedge c\right) \vee c^{-}\right)=x(c), \quad \text { or } \\
& x^{-1}(u(x)) \wedge c \leqslant c^{-} \quad \text { and so } \quad u^{\prime}(x)=x\left(\left(x^{-1}(u(x)) \wedge c\right) \vee c^{-}\right)=x\left(c^{-}\right) \text {. }
\end{aligned}
$$

Moreover, note that $u(x)=x\left(u\left(\operatorname{id}_{M}\right)\right)$ implies $u^{\prime}(x)=x\left(u^{\prime}\left(\operatorname{id}_{M}\right)\right)$.

Also observe that in case End $\underline{\mathbf{M}} \neq$ Aut $\underline{\mathbf{M}}$, we must impose $\left(c, c^{-}\right) \in \kappa$, by Proposition 3.1.

Thus we have just proved the following result.

PROPOSITION 3.4. Let $U$ be a globally minimal failset whose minimal elements are automorphisms. If $\underline{\mathbf{M}}$ is a lattice-structured algebra, then there exist a map $u:$ End $\underline{\mathbf{M}} \rightarrow M$ and $a, b \in M$ such that the following conditions hold:

(a) $U=$ Fail $_{g}(u)$, for some $g \in U_{\min }$;

(b) $u\left(\mathrm{id}_{M}\right)=a, u(g)=g(b)$;

(c) $\forall x \in U_{\min }, u(x) \in\{x(a), x(b)\}$;

(d) $(a, b) \in \kappa,[a, b] \succ \triangle_{M}$ and either $a \in \mathscr{J}(\underline{\mathbf{M}})$, with $b=a^{-}$, or $b \in \mathscr{J}(\underline{\mathbf{M}})$, with $a=b^{-}$.

Take a map $u:$ End $\underline{\mathbf{M}} \rightarrow M$ and $a, b \in M$ to satisfy the conditions of Proposition 3.4, and define a map $v:$ End $\underline{\mathbf{M}} \rightarrow M$ by $v(x)=u(x \circ g)$. Observe that $U$ is $\operatorname{Fail}_{g^{-1}}(v)$ and the following conditions hold:

(a) $v\left(\mathrm{id}_{M}\right)=g(b)$ and $v\left(g^{-1}\right)=a=g^{-1}(g(a))$;

(b) $[a, b]>\Delta_{M} \Rightarrow[g(a), g(b)]>\Delta_{M}$;

(c) $g$ preserves join-irreducible elements of $\underline{\mathbf{M}}$ and $(g(c))^{-}=g\left(c^{-}\right)$, for every $c \in \mathscr{J}(\underline{\mathbf{M}})$

(d) $g$ preserves $\kappa$. 
We may now reformulate Proposition 3.4 as presented below.

PROPOSITION 3.5. Let $U$ be a globally minimal failset whose minimal elements are automorphisms. If $\underline{\mathbf{M}}$ is a lattice-structured algebra, then there exist a map $u:$ End $\underline{\mathbf{M}} \rightarrow M$ and $a \in \mathscr{J}(\underline{\mathrm{M}})$ such that the following conditions hold:

(a) $U=$ Fail $_{g}(u)$, for some $g \in U_{\min }$;

(b) $u\left(\mathrm{id}_{M}\right)=a, u(g)=a^{-}$;

(c) $\forall x \in U_{\min }, u(x) \in\left\{x(a), x\left(a^{-}\right)\right\}$;

(d) $\left(a, a^{-}\right) \in \kappa$ and $\left[a, a^{-}\right] \succ \Delta_{M}$.

The next result tells us that the failsets Fail $_{g}(u)$ only depend on the set of automorphisms $x$ of $\underline{\mathbf{M}}$ on which $u$ does not take the value $x\left(u\left(\mathrm{id}_{M}\right)\right)$, and on the cover $[a, b]$ of $\Delta_{M}$ we choose; it does not depend on the pair $\left(a^{\prime}, b^{\prime}\right)$ we take among those that generate $[a, b]$.

Proposition 3.6. Let $u:$ End $\underline{\mathrm{M}} \rightarrow M$ be a map such that Fail $_{g}(u)$ is a failset of $g \in$ Aut $\underline{\mathbf{M}}$ and suppose that there exist $a, b \in M$ such that $u(x) \in\{x(a), x(b)\}$, for every $x \in$ End $\underline{\mathbf{M}}$. For every $a^{\prime}, b^{\prime} \in M$, if $\left[a^{\prime}, b^{\prime}\right]=[a, b]$ then $\operatorname{Fail}_{g}(u)=\operatorname{Fail}_{g}\left(u^{\prime}\right)$, where $u^{\prime}:$ End $\underline{\mathbf{M}} \rightarrow M$ is the map defined by

$$
u^{\prime}(x)= \begin{cases}x\left(a^{\prime}\right) & \text { if } u(x)=x(a) \\ x\left(b^{\prime}\right) & \text { otherwise. }\end{cases}
$$

PROOF. Let $s$ be a binary algebraic relation on $\underline{\mathbf{M}}$. For every $x, y \in$ End $\underline{\mathbf{M}}$, we have that

$$
\begin{aligned}
(x, y) & \in s \wedge(x(a), y(b)) \notin s \\
& \Longleftrightarrow \Delta_{M} \subseteq(x \times y)^{-1}(s) \wedge(a, b) \notin(x \times y)^{-1}(s) \\
& \Longleftrightarrow \Delta_{M} \subseteq(x \times y)^{-1}(s) \wedge\left[a^{\prime}, b^{\prime}\right]=[a, b] \nsubseteq(x \times y)^{-1}(s) \\
& \Longleftrightarrow \Delta_{M} \subseteq(x \times y)^{-1}(s) \wedge\left(a^{\prime}, b^{\prime}\right) \notin(x \times y)^{-1}(s) \\
& \Longleftrightarrow(x, y) \in s \wedge\left(x\left(a^{\prime}\right), y\left(b^{\prime}\right)\right) \notin s .
\end{aligned}
$$

Therefore, $(x, y)$ witnesses $s \in$ Fail $_{g}(u)$ if and only if $(x, y)$ witnesses $s \in$ Fail $_{g}\left(u^{\prime}\right)$. Thus Fail $g(u)=$ Fail $_{g}\left(u^{\prime}\right)$.

The number of atoms in the lattice of congruences on $\underline{\mathbf{M}}$, Con $\underline{\mathbf{M}}$, is equal to the number of sets $\left\{\left[a, a^{-}\right],\left[a^{-}, a\right]\right\}$, where $a \in \mathscr{J}(\underline{\mathbf{M}})$ and $\left[a, a^{-}\right] \succ \Delta_{M}$ in $\mathbb{S}\left(\underline{\mathbf{M}}^{2}\right)$ (see [6, Proposition 1.9]). Thus, the number of choices of maps $u$ is as big as the number of atoms of Con $\underline{\mathbf{M}}$ contained in $\kappa$ if End $\underline{\mathbf{M}} \neq$ Aut $\underline{\mathbf{M}}$, and the number of atoms of $\operatorname{Con} \underline{\mathbf{M}}$ otherwise. Naturally, the simplest situation we may consider is when the congruence lattice has only one atom, that is, when $\underline{\mathbf{M}}$ is subdirectly irreducible. Besides, this is 
also the case for many of the globally minimal failsets we know, among which there are those we mention at the beginning of this section.

Henceforward we consider that $\underline{\mathbf{M}}$ is a subdirectly irreducible lattice-structured algebra. Consequently, the trivial relation $\Delta_{M}$ has at most two covers $\left[a, a^{-}\right],\left[a^{-}, a\right]$ in $\$\left(\underline{\mathbf{M}}^{2}\right)$, given by a non-zerojoin-irreducible element $\boldsymbol{a}$ of $\underline{\mathbf{M}}$. We also assume that, in case End $\underline{\mathbf{M}} \neq$ Aut $\underline{\mathbf{M}}$, the congruence $\kappa$ is not $\Delta_{M}$ since this is a necessary condition for having a globally minimal failset whose minimal elements are automorphisms, according to Proposition 3.1.

We show that every maximal proper subgroup of Aut $\underline{\mathbf{M}}$ determines a globally minimal failset and, besides these, there are no other globally minimal failsets whose minimal elements are automorphisms.

The case $\left[a, a^{-}\right]=\left[a^{-}, a\right]$. We first consider that $\Delta_{M}$ has a unique cover in $\$\left(\underline{M}^{2}\right)$, that is, $\left[a, a^{-}\right]=\left[a^{-}, a\right]$. Therefore every automorphism $g$ of $\underline{\mathbf{M}}$ has a unique cover $\left[g \cup\left\{\left(a, g\left(a^{-}\right)\right)\right\}\right]$. If, for every proper subgroup $H$ of Aut $\underline{\mathbf{M}}$, we could define a failset Fail $_{g}(u)$ whose minimal elements were the automorphisms not contained in $H$, in such a way that their covers would be preserved by the map $u$, then we would expect that the globally minimal failsets were exactly the complements in Aut $\underline{\mathbf{M}}$ of the maximal proper subgroups of Aut $\underline{\mathbf{M}}$.

PROPOSITION 3.7. Let $H$ be a maximal proper subgroup of Aut $\underline{\mathrm{M}}$. Then the set Aut $\underline{\mathbf{M}} \backslash H$ is a globally minimal failset.

Proof. Define a map $u:$ End $\underline{\mathbf{M}} \rightarrow M$ by

$$
u(x)= \begin{cases}x(a) & \text { if } x \notin \text { Aut } \underline{\mathbf{M}} \text { or } x \in H ; \\ x\left(a^{-}\right) & \text {otherwise. }\end{cases}
$$

Let $g \in$ Aut $\underline{\mathbf{M}} \backslash H$. The pair $\left(\operatorname{id}_{M}, g\right.$ ) witnesses $g \in$ Fail $_{g}(u)$. We claim that this failset of $g$ is Aut $\underline{\mathbf{M}} \backslash H$. Take $s \in \operatorname{Fail}_{g}(u)$. Notice that if End $\underline{\mathbf{M}} \neq$ Aut $\underline{\mathbf{M}}$ then $f(a)=f\left(a^{-}\right)$, for every $f \in \operatorname{End} \underline{\mathbf{M}} \backslash$ Aut $\underline{\mathbf{M}}$. Hence there exist $x, y \in$ Aut $\underline{\mathbf{M}}$ such that $(x, y) \in s$ and either $\left(x(a), y\left(a^{-}\right)\right) \notin s$ or $\left(x\left(a^{-}\right), y(a)\right) \notin s$. Then $\left(a, a^{-}\right) \notin$ $(x \times y)^{-1}(s)$ since $\Delta_{M} \subseteq(x \times y)^{-1}(s)$ and $\left[a, a^{-}\right]=\left[a^{-}, a\right]$. Consequently we must have $(x \times y)^{-1}(s)=\Delta_{M}$ and therefore $s=y \circ x^{-1} \notin H$. Thus Fail ${ }_{g}(u)=$ Aut $\underline{\mathbf{M}} \backslash H$ and, by the maximality of $H$ as a proper subgroup of Aut $\underline{\mathbf{M}}$, we have that the failset Aut $\underline{\mathbf{M}} \backslash H$ is indeed a globally minimal failset.

PROPOSITION 3.8. Let $U$ be a globally minimal failset whose minimal elements are automorphisms. Then $U=U_{\min }$ and the subgroup Aut $\underline{\mathbf{M}} \backslash U$ is a maximal proper subgroup of Aut $\underline{\text { M. }}$ 
Proof. Take $H$ to be a maximal proper subgroup of Aut $\underline{\mathbf{M}}$ containing the proper subgroup Aut $\underline{\mathbf{M}} \backslash U_{\min }$. By the last result, Aut $\underline{\mathbf{M}} \backslash H$ is a globally minimal failset and, since it is contained in $U_{\min }$, we necessarily have that $U=\operatorname{Aut} \underline{\mathbf{M}} \backslash H=U_{\min }$.

Thus the following theorem comes immediately.

THEOREM 3.9. If $\Delta_{M}$ has a unique cover in $\mathbb{S}\left(\underline{\mathbf{M}}^{2}\right)$, then there are globally minimal failsets whose minimal elements are automorphisms and they are precisely the complements in Aut $\underline{\mathbf{M}}$ of maximal proper subgroups of Aut $\underline{\mathbf{M}}$.

The case $\left[a, a^{-}\right] \neq\left[a^{-}, a\right]$. Next we consider the case $\left[a, a^{-}\right] \neq\left[a^{-}, a\right]$. Notice that two situations may occur. Either $\left[a, a^{-}\right]$is preserved by every $x \in$ Aut $\underline{\mathbf{M}}$, or $x\left(\left[a, a^{-}\right]\right)=\left[a^{-}, a\right]$, for some $x \in$ Aut $\underline{\mathbf{M}}$.

Denote by $S_{a a^{-}}$the set of subalgebras of $\underline{\mathbf{M}}^{2}$ whose intersection with $\left[a, a^{-}\right]$is $\Delta_{M}$ and denote by $S_{a^{-} a}$ the set of subalgebras of $\underline{\mathbf{M}}^{2}$ whose intersection with $\left[a^{-}, a\right] \mathrm{i}$ $\Delta_{M}$. Note that $S_{a^{-} a}$ is precisely the set of algebras $\mathbf{s}^{\smile}$, where $\mathbf{s} \in S_{a a^{-}}$, and $\Delta_{M}$ i the smallest subalgebra of $\underline{\mathbf{M}}^{2}$ in $S_{a a^{-}} \cup S_{a^{-} a^{-}}$. It follows from the proposition belo' that $\mathrm{id}_{M}$ is the unique automorphism whose graph is contained in a subalgebra r $S_{a a^{-}} \cup S_{a^{-} a}$.

PROPOSITION 3.10. Let $\mathbf{S}$ be a subalgebra of $\underline{\mathrm{M}}^{2}$ and suppose that $\Delta_{M} \subseteq s$ but $\left(a, a^{-}\right) \notin s$. Then $\mathrm{id}_{M}$ is the unique automorphism whose graph is contained in $s$.

PROOF. Let $f \in$ Aut $\underline{\mathbf{M}}$ and suppose that graph $f \subseteq s$. Note that $f=\operatorname{id}_{M}$ if $f(c)=c$, for every $c \in \mathscr{J}(\underline{\mathbf{M}})$. Let $c \in \mathscr{J}(\underline{\mathbf{M}})$. If $f(c) \neq c$ then $c$ and $f(c)$ are non-comparable (see [6, Propositions 1.1-1.2]). But then we have $(c, f(c) \wedge c)=$ $(c, f(c)) \wedge(c, c) \in s$ and $\left(c, c^{-}\right)=(c, f(c) \wedge c) \vee\left(c^{-}, c^{-}\right) \in s$ (recall that $f(c) \wedge c<c$ implies $\left.f(c) \wedge c \leqslant c^{-}\right)$. Similarly, we also have that $\left(c^{-}, c\right) \in s$ since $c$ and $f^{-1}(c)$ are non-comparable and $\left(f^{-1}(c), c\right) \in s$. Observe that both $\left[c, c^{-}\right],\left[c^{-}, c\right]$ properly contain $\Delta_{M}$ and they are both contained in $s$. Also $\left(a, a^{-}\right) \in\left[c, c^{-}\right]$if and only if $\left(a^{-}, a\right) \in\left[c^{-}, c\right]$. Then $\left(a, a^{-}\right) \in s$ and we get a contradiction.

For every proper subgroup $H$ of Aut $\underline{\boldsymbol{M}}$, denote by $U_{H}^{a a^{-}}$the set

$$
\left((x \times y)(r),((x \times y)(r))^{-}|x \in H \wedge y \in A u t \underline{M}| H \wedge r \in S_{a a^{-}}\right\},
$$

and by $U_{H}^{a^{-} a}$ the set

$$
\left\{(x \times y)(r),((x \times y)(r))^{\smile} \mid x \in H \wedge y \in \text { Aut } \underline{\mathbf{M}} \backslash H \wedge \mathbf{r} \in S_{a^{-} a}\right\} .
$$

Next we prove that the sets $U_{H}^{a a^{-}}$and $U_{H}^{a^{-} a}$ are failsets of its minimal elements: the automorphisms that do not belong to $H$. 
PROPOSITION 3.11. Let $H$ be a proper subgroup of Aut $\underline{\mathbf{M}}$ and let $g \in \operatorname{Aut} \underline{\mathbf{M}} \backslash H$. Then the sets $U_{H}^{a a^{-}}$and $U_{H}^{a^{-} a}$ are respectively the failsets of $g$ : Fail $_{g}\left(u_{a a^{-}}\right)$, where $u_{a a^{-}}:$End $\underline{\mathbf{M}} \rightarrow M$ is the map defined by

$$
u_{a a^{-}}(x)= \begin{cases}x\left(a^{-}\right) & \text {if } x \in \text { Aut } \underline{\mathbf{M}} \backslash H \\ x(a) & \text { otherwise, }\end{cases}
$$

and Fail $_{g}\left(u_{a^{-a}}\right)$, where $u_{a^{-a}}:$ End $\underline{\mathbf{M}} \rightarrow M$ is the map defined by

$$
u_{a^{-} a}(x)= \begin{cases}x(a) & \text { if } x \in \text { Aut } \underline{\mathbf{M}} \backslash H \\ x\left(a^{-}\right) & \text {otherwise. }\end{cases}
$$

PROOF. Clearly Fail ${ }_{g}\left(u_{a a^{-}}\right)$is a failset of $g$. Take $\mathbf{r} \in S_{a a^{-}}$and take $x \in H$ and $y \in \operatorname{Aut} \underline{\mathbf{M}} \backslash H$. Then $(x, y)$ witnesses $(x \times y)(r) \in \operatorname{Fail}_{g}\left(u_{a a^{-}}\right)$because $\left(a, a^{-}\right) \notin r$. Recall that a failset contains a relation $s$ if and only if it contains $s^{\smile}$, and therefore $(x \times y)(r),((x \times y)(r))^{\smile} \in \operatorname{Fail}_{g}\left(u_{a a^{-}}\right)$. Thus $U_{H}^{a a^{-}} \subseteq \operatorname{Fail}_{g}\left(u_{a a^{-}}\right)$. Now take $s \in$ Fail $_{g}\left(u_{a a^{-}}\right)$. Then there exist $x, y \in$ End $\underline{\mathbf{M}}$ such that $(x, y) \in s$ and $\left(u_{a a^{-}}(x), u_{a a^{-}}(y)\right) \notin s$. Since $s \in U_{H}^{a a^{-}}$if and only if $s^{\smile} \in U_{H}^{a a^{-}}$, we may assume that $\left(u_{a a^{-}}(x), u_{a a^{-}}(y)\right)=\left(x(a), y\left(a^{-}\right)\right)$. Then $x \in H, y \in$ Aut $\underline{\mathbf{M}} \backslash H$ (recall that $\left(a, a^{-}\right) \in \kappa$ in case End $\left.\underline{\mathbf{M}} \neq \operatorname{Aut} \underline{\mathbf{M}}\right)$ and $\left(a, a^{-}\right) \notin(x \times y)^{-1}(s)$. Since $(x \times y)^{-1}(\mathbf{s}) \in S_{a a^{-}}$, we have $s=(x \times y)\left((x \times y)^{-1}(s)\right) \in U_{H}^{a a^{-}}$. Thus $U_{H}^{a a^{-}}=$Fail $_{g}\left(u_{a a^{-}}\right)$.

PROPOSITION 3.12. Let $H$ be a proper subgroup of Aut $\underline{M}$. If $U$ is a globally minimal failset contained in one of the failsets $U_{H}^{a a^{-}}$and $U_{H}^{a^{-a}}$, then $U_{\min }$ is a subset of Aut $\underline{\mathbf{M}} \backslash H$; in case $H$ is a maximal proper subgroup of Aut $\underline{\mathbf{M}}$, we have that $U_{\min }=$ Aut $\underline{\mathbf{M}} \backslash H$.

Proof. Let $U$ be a globally minimal failset and suppose $U \subseteq U_{H}^{a a^{-}}$. Take $x \in H$, $y \in$ Aut $\underline{\mathbf{M}} \backslash H$ and $\mathbf{r} \in S_{a a^{-}}$such that $(x \times y)(r) \in U$. By Proposition 3.10, $\mathrm{id}_{M}$ is the unique automorphism whose graph is contained in $r$. But then $r \notin U_{H}^{a a-}$ and thereby $r \notin U$. It follows that $y \in U$ because $x \notin U$. Hence graph $\left(y \circ x^{-1}\right) \subseteq(x \times y)(r)$ and $y \circ x^{-1} \in U$. Therefore $U_{\min } \subseteq$ Aut $\underline{\mathbf{M}}$. So $U_{\min } \subseteq$ Aut $\underline{\mathbf{M}} \cap U_{H}^{a a^{-}}=$Aut $\underline{\mathbf{M}} \backslash H$. For the rest of the proof, only notice that $\mathrm{Aut} \underline{\mathrm{M}} \backslash U_{\min }$ is a proper subgroup of Aut $\underline{\mathbf{M}}$ that contains $H$.

Note that

$$
(x \times y)(r)=\left(\operatorname{id}_{M} \times\left(y \circ x^{-1}\right)\right)((x \times x)(r))
$$

and

$$
\left(\left(\operatorname{id}_{M} \times y\right)(r)\right)^{\smile}=\left(\operatorname{id}_{M} \times y^{-1}\right)\left((y \times y)\left(r^{\smile}\right)\right),
$$


for every $x, y \in$ Aut $\underline{\mathbf{M}}$ and $\mathbf{r} \in S_{a a^{-}} \cup S_{a^{-} a}$. If $x, y \in$ Aut $\underline{\mathbf{M}}$ satisfy $x\left(\left[a, a^{-}\right]\right)=$ $\left[a, a^{-}\right]$and $y\left(\left[a, a^{-}\right]\right)=\left[a, a^{-}\right]$, then

$$
(x \times x)(\mathbf{r}) \in S_{a a^{-}} \Longleftrightarrow \mathbf{r} \in S_{a a^{-}} \Longleftrightarrow \mathbf{r}^{\sim} \in S_{a^{-} a} \Longleftrightarrow(y \times y)\left(\mathbf{r}^{\smile}\right) \in S_{a^{-} a} .
$$

Hence suppose that $x\left(\left[a, a^{-}\right]\right)=\left[a, a^{-}\right]$, for every $x \in$ Aut $\underline{M}$. We have

$$
\begin{aligned}
U_{H}^{a a^{-}} & =\left\{\left(\operatorname{id}_{M} \times x\right)(r),\left(\left(\operatorname{id}_{M} \times x\right)(r)\right)^{\smile} \mid x \in \text { Aut } \underline{\mathbf{M}} \backslash H \wedge \mathbf{r} \in S_{a a^{-}}\right\} \\
& =\left\{\left(\operatorname{id}_{M} \times x\right)(r) \mid x \in \text { Aut } \underline{\mathbf{M}} \backslash H \wedge \mathbf{r} \in S_{a a^{-}} \cup S_{a^{-a}}\right\} \\
& =\left\{\left(\operatorname{id}_{M} \times x\right)(r),\left(\left(\operatorname{id}_{M} \times x\right)(r)\right)^{\smile} \mid x \in \text { Aut } \underline{\mathbf{M}} \backslash H \wedge \mathbf{r} \in S_{a^{-} a}\right\}=U_{H}^{a^{-} a},
\end{aligned}
$$

for every proper subgroup $H$ of Aut $\underline{\mathbf{M}}$. So we simply denote $U_{H}^{a a^{-}}$by $U_{H}$. We show that the globally minimal failsets whose minimal elements are automorphisms of $\underline{\mathbf{M}}$ are exactly the sets $U_{H}$, where $H$ is a maximal proper subgroup of Aut $\underline{\mathbf{M}}$.

PROPOSITION 3.13. Let $U$ be a globally minimal failset whose minimal elements are automorphisms. If $x\left(\left[a, a^{-}\right]\right)=\left[a, a^{-}\right]$, for every $x \in$ Aut $\underline{\mathbf{M}}$, then $U=U_{H}$, where $H=$ Aut $\underline{\mathbf{M}} \backslash U_{\min }$.

PROOF. Recall that $U_{H}$ is a failset of every $g \in U_{\min }$, by Proposition 3.11. Since $U$ is a minimal failset of every $g \in U_{\min }$, we only need to prove that $U_{H} \subseteq U$. By Propositions 3.5 and 3.6, there exist $g \in U_{\min }$ and a map $u:$ End $\underline{\mathbf{M}} \rightarrow M$ such that $U=\operatorname{Fail}_{g}(u)$ and $u(x) \in\left\{x(a), x\left(a^{-}\right)\right\}$, for every $x \in$ End $\underline{\mathbf{M}}$. Take $\mathbf{r} \in S_{a a^{-}}$and $f \in U_{\min }$. There is $x \in$ Aut $\underline{\mathbf{M}}$ such that $u(f \circ x) \neq f(u(x))$. If $(u(x), u(f \circ x)) \notin\left(\mathrm{id}_{M} \times f\right)(r)$ then $\left(\mathrm{id}_{M} \times f\right)(r) \in U$. If $(u(x), u(f \circ x)) \in$ $\left(\operatorname{id}_{M} \times f\right)(r)$ then $(u(x), u(f \circ x))=\left(x\left(a^{-}\right),(f \circ x)(a)\right)$, or otherwise

$$
\left(a, a^{-}\right) \in\left[x(a), x\left(a^{-}\right)\right]=\left[u(x), f^{-1}(u(f \circ x))\right] \subseteq r .
$$

Since $u(x)=x\left(a^{-}\right)$, there must exist $k \geqslant 2$ such that $u\left(f^{k} \circ x\right)=\left(f^{k} \circ x\right)\left(a^{-}\right)$. Moreover, we may take $k$ to satisfy $u\left(f^{k-1} \circ x\right)=\left(f^{k-1} \circ x\right)(a)$ too. Then we get

$$
\left(u\left(f^{k-1} \circ x\right), u\left(f^{k} \circ x\right)\right)=\left(\left(f^{k-1} \circ x\right)(a),\left(f^{k} \circ x\right)\left(a^{-}\right)\right) \notin\left(\operatorname{id}_{M} \times f\right)(r)
$$

because $\left[\left(f^{k-1} \circ x\right)(a),\left(f^{k-1} \circ x\right)\left(a^{-}\right)\right]=\left[a, a^{-}\right]$and $\left(a, a^{-}\right) \notin r$.

Thus $\left(\mathrm{id}_{M} \times f\right)(r) \in U$.

THEOREM 3.14. Let $\left[a, a^{-}\right]$and $\left[a^{-}, a\right]$ be the unique two covers of $\triangle_{M}$ in $\$\left(\underline{M}^{2}\right)$ and suppose that $x\left(\left[a, a^{-}\right]\right)=\left[a, a^{-}\right]$, for every $x \in$ Aut $\underline{\mathbf{M}}$. Then a subset $U$ of $\mathbb{S}\left(\underline{\mathbf{M}}^{2}\right)$ is a globally minimal failset whose minimal elements are automorphisms if and only if $U=U_{H}$, for some maximal proper subgroup $H$ of Aut $\underline{\mathbf{M}}$. 
Proof. Note that $U_{H^{\prime}} \subseteq U_{H}$ whenever $H$ and $H^{\prime}$ are proper subgroups of Aut $\underline{\mathrm{M}}$ such that $H \subseteq H^{\prime}$. Hence, if $U$ is a globally minimal failset with $U_{\min } \subseteq$ Aut $\underline{\mathbf{M}}$, it is immediate that $U=U_{H}$ and $H=$ Aut $\underline{\mathbf{M}} \backslash U_{\min }$ is a maximal proper subgroup of Aut $\underline{\mathrm{M}}$, by applying Propositions 3.11 and 3.13. Now take $H$ to be a maximal proper subgroup of Aut $\underline{\mathbf{M}}$. Recall that Proposition 3.11 tell us that $U_{H}$ is a failset of each of its automorphisms. If $U$ is a globally minimal failset contained in $U_{H}$, then, by Proposition 3.12, $U_{\min }=$ Aut $\underline{\mathbf{M}} \backslash H$. Now, from the previous proposition, it follows that $U_{H}=U$ is a globally minimal failset.

As an application, take the example $\underline{\mathbf{M}}=\mathbf{P}_{m, n}$ studied in [7]. We have that $\theta\left(d_{2}, d_{1}\right)=\overline{\text { id }}$ is the unique atom of $\operatorname{Con} \mathbf{P}_{m, n},\left[d_{2}, d_{1}\right]=\left(\mathrm{id}^{\leqslant}\right)^{\smile}$ and $S_{d_{2} d_{1}}=\left\{\mathrm{id}\right.$, id $\left.{ }^{\leqslant}\right\}$. Also End $\mathbf{P}_{m, n}=$ Aut $\mathbf{P}_{m, n}$ and $x\left(\left[d_{2}, d_{1}\right]\right)=\left[d_{2}, d_{1}\right]$, for every $x \in$ Aut $\mathbf{P}_{m, n}$. Hence we may apply Theorem 3.14 and, as we expected, the globally minimal failsets $U_{H}$ are the sets $W_{H}=K \cup\left\{g^{\leqslant},\left(g^{\leqslant}\right)^{-} \mid g \in K\right\}$, where $K=$ Aut $\mathbf{P}_{m, n} \backslash H$.

Finally consider that $x\left(\left[a, a^{-}\right]\right)=\left[a^{-}, a\right]$, for some $x \in$ Aut $\underline{\mathbf{M}}$. Observe that $H_{a, a^{-}}:=\left\{x \in\right.$ Aut $\left.\underline{\mathbf{M}} \mid x\left(\left[a, a^{-}\right]\right)=\left[a, a^{-}\right]\right\}$is a proper subgroup of Aut $\underline{\mathbf{M}}$. Moreover, $H_{a, a^{-}}$is a maximal proper subgroup of Aut $\underline{\mathbf{M}}$ :

Take $H$ to be a proper subgroup of Aut $\underline{\mathbf{M}}$ such that $H_{a, a^{-}} \subseteq H$. Take $y \notin H$. For every $x \in H$, we have $y, y \circ x \notin H$ and then $y, y \circ x \notin H_{a, a^{-}}$. Hence we have $y\left(\left[a, a^{-}\right]\right)=\left[a^{-}, a\right]=(y \circ x)\left(\left[a, a^{-}\right]\right)$which implies $x\left(\left[a, a^{-}\right]\right)=\left[a, a^{-}\right]$and so $x \in H_{a, a^{-}}$.

From Proposition 3.11 it follows that the sets $U_{H_{a, a^{-}}}^{a a^{-}}$and $U_{H_{a, a^{-}}}^{a^{-} a}$ are failsets of their automorphisms. Now, for every $y \in$ Aut $\underline{\mathbf{M}} \backslash H_{a, a^{-}}$and $\mathbf{r} \in S_{a a^{-}} \cup S_{a^{-a} a}$, we have that

$$
\mathbf{r} \in S_{a a^{-}} \Longleftrightarrow \mathbf{r}^{\smile} \in S_{a^{-} a} \Longleftrightarrow(y \times y)\left(\mathbf{r}^{\smile}\right) \in S_{a a^{-}} .
$$

Hence

$$
\begin{aligned}
& U_{H_{a, a^{-}}}^{a a^{-}}=\left\{\left(\mathrm{id}_{M} \times x\right)(r) \mid x \in \text { Aut } \underline{\mathbf{M}} \backslash H_{a, a^{-}} \wedge \mathbf{r} \in S_{a a^{-}}\right\} \text {and } \\
& U_{H_{a, a^{-}}}^{a^{-a}}=\left\{\left(\operatorname{id}_{M} \times x\right)(r) \mid x \in \text { Aut } \underline{\mathbf{M}} \backslash H_{a, a^{-}} \wedge \mathbf{r} \in S_{a^{-a}}\right\}
\end{aligned}
$$

PROPOSITION 3.15. The intersection of $U_{H_{a, a^{-}}}^{a a^{-}}$and $U_{H_{a, a^{-}}}^{a^{-a}}$ is Aut $\underline{\mathbf{M}} \backslash H_{a, a^{-}}$.

Proof. Let $x \in$ Aut $\underline{\mathbf{M}} \backslash H_{a, a^{-}}$and let $\mathbf{r} \in S_{a a^{-}}$. Suppose that $\left(\operatorname{id}_{M} \times x\right)(r)=$ $\left(\operatorname{id}_{M} \times y\right)(s)$, for some $y \in$ Aut $\underline{\mathbf{M}} \backslash H_{a, a^{-}}$and $s \in S_{a^{-} a}$. Since $\mathrm{id}_{M}$ is the unique automorphism whose graph is contained in $r$, we must have that $x=y$ and therefore $r=s$. Thus $r \in S_{a a^{-}} \cap S_{a^{-a}}=\left\{\Delta_{M}\right\}$.

Now take $U$ to be a globally minimal failset contained in $U_{H_{a, a^{-}}}^{a a^{-}}$. By Proposition 3.12, the set $U_{\min }$ is Aut $\underline{\mathbf{M}} \backslash H_{a, a^{-}}$and, by Proposition 3.15, $\left(\mathrm{id}_{M} \times g\right)\left(\left[a, a^{-}\right]\right) \notin$ $U$, for every $g \in U_{\min }$. This suggests that we should analyse a more general case, 
where $U$ is a globally minimal failset whose minimal elements are automorphisms and such that either $\left(\mathrm{id}_{M} \times g\right)\left(\left[a, a^{-}\right]\right) \notin U$ or $\left(\mathrm{id}_{M} \times g\right)\left(\left[a^{-}, a\right]\right) \notin U$, for some $g \in U_{\min }$.

Hence, take $U$ to be a globally minimal failset such that $U_{\min } \subseteq$ Aut $\underline{M}$ and suppose that there exists $g \in U_{\min }$ such that $\left(\operatorname{id}_{M} \times g\right)\left(\left[a, a^{-}\right]\right) \notin U$. Consider the map $u:$ End $\underline{\mathbf{M}} \rightarrow M$ defined as follows

$$
u(x)= \begin{cases}x(a) & \text { if }\left(\mathrm{id}_{M} \times x\right)\left(\left[a, a^{-}\right]\right) \in U \text { or } x \notin U ; \\ x\left(a^{-}\right) & \text {otherwise. }\end{cases}
$$

LEMMA 3.16. The globally minimal failset $U$ is Fail $_{g}(u)$.

Proof. By Propositions 3.4 and 3.6, there exist a map $v:$ End $\underline{\mathbf{M}} \rightarrow M$ and $f \in U_{\min }$ such that $U=\operatorname{Fail}_{f}(v)$ and $v(x) \in\left\{x(a), x\left(a^{-}\right)\right\}$, for every $x \in$ End $\underline{\mathbf{M}}$. Note that $g \in$ Fail $_{g}(u)$ is witnessed by $\left(\operatorname{id}_{M}, g\right.$ ). Since $U$ is a minimal failset of $g$, we only need to prove that $\operatorname{Fail}_{g}(u) \subseteq U$. Take $s \in$ Fail $_{g}(u)$. We may assume that there exist $x, y \in$ Aut $\underline{\mathbf{M}}$ such that $y \circ x^{-1} \subseteq s$ and $(u(x), u(y))=\left(x(a), y\left(a^{-}\right)\right) \notin s$. We are going to consider two situations.

(i) Suppose that $x \notin U$. Then we have

$$
(x \times y)\left(\left[a, a^{-}\right]\right)=\left(x \times \operatorname{id}_{M}\right)\left(\left(\operatorname{id}_{M} \times y\right)\left(\left[a, a^{-}\right]\right)\right) \notin U
$$

because $\left(\operatorname{id}_{M} \times y\right)\left(\left[a, a^{-}\right]\right) \notin U$. Since $y \circ x^{-1} \in U$, there exists $z \in$ Aut $\underline{\mathbf{M}}$ such that $\left(v(z), v\left(y \circ x^{-1} \circ z\right)\right) \notin y \circ x^{-1}$. But we have

$$
\left(v(z), v\left(y \circ x^{-1} \circ z\right)\right) \in(x \times y)\left(\left[a, a^{-}\right]\right)=\left(\operatorname{id}_{M} \times\left(y \circ x^{-1}\right)\right)\left(\left[x(a), x\left(a^{-}\right)\right]\right) .
$$

Consequently $\left[v(z),\left(y \circ x^{-1}\right)^{-1}\left(v\left(y \circ x^{-1} \circ z\right)\right)\right]=\left[x(a), x\left(a^{-}\right)\right]$. We also have $\left(x(a), x\left(a^{-}\right)\right) \notin\left(\mathrm{id}_{M} \times\left(y \circ x^{-1}\right)\right)^{-1}(s)$ because $\left(x(a), y\left(a^{-}\right)\right) \notin s$, and so

$$
\left(v(z),\left(y \circ x^{-1}\right)^{-1}\left(v\left(y \circ x^{-1} \circ z\right)\right)\right) \notin\left(\mathrm{id}_{M} \times\left(y \circ x^{-1}\right)\right)^{-1}(s) .
$$

Hence $s \in U$ is witnessed by $\left(z, y \circ x^{-1} \circ z\right)$.

(ii) Suppose that $x \in U$ and $\left(\operatorname{id}_{M} \times x\right)\left(\left[a, a^{-}\right]\right) \in U$. On the one hand, we may take $z \in$ Aut $\underline{\mathbf{M}}$ to be such that $(v(z), v(x \circ z)) \notin\left(\mathrm{id}_{M} \times x\right)\left(\left[a, a^{-}\right]\right)$and then $\left[v(z), x^{-1}(v(x \circ z))\right]=\left[a^{-}, a\right]$. On the other hand, $(v(z), v(y \circ z)) \in\left(\operatorname{id}_{M} \times y\right)\left(\left[a, a^{-}\right]\right)$ because $\left(\operatorname{id}_{M} \times y\right)\left(\left[a, a^{-}\right]\right) \notin U$, and thus

$$
\text { either } v(y \circ z)=y(v(z)) \text { or }\left[v(z), y^{-1}(v(y \circ z))\right]=\left[a, a^{-}\right] .
$$

If $v(y \circ z)=y(v(z))$ then

$$
\left[a, a^{-}\right]=\left[x^{-1}(v(x \circ z)), v(z)\right]=\left[x^{-1}(v(x \circ z)), y^{-1}(v(y \circ z))\right] .
$$


Note that $\left(a, a^{-}\right) \notin(x \times y)^{-1}(s)$ and $\Delta_{M} \subseteq(x \times y)^{-1}(s)$. Therefore

$$
\left(x^{-1}(v(x \circ z)), y^{-1}(v(y \circ z))\right) \notin(x \times y)^{-1}(s)
$$

which implies $(v(x \circ z), v(y \circ z)) \notin s$.

If $\left[v(z), y^{-1}(v(y \circ z))\right]=\left[a, a^{-}\right]$then we have

$$
v(x \circ z)=x \circ z(a) \Longleftrightarrow v(z)=z\left(a^{-}\right) \Longleftrightarrow v(y \circ z)=y \circ z(a)
$$

because $\left[v(z), x^{-1}(v(x \circ z))\right]=\left[a^{-}, a\right]$, and so

$$
\left[a^{-}, a\right]=\left[v(z), x^{-1}(v(x \circ z))\right]=\left[v(z), y^{-1}(v(y \circ z))\right]=\left[a, a^{-}\right] .
$$

Thus $s \in U$.

LEMMA 3.17. For every $x \in$ Aut $\underline{\mathbf{M}}$ condition $u(x)=x\left(a^{-}\right)$holds if and only if $x\left(\left[a, a^{-}\right]\right)=\left[a^{-}, a\right]$ and $x \in U$.

Proof. First we prove that for any $x \in$ Aut $\underline{\mathrm{M}}$, if $u(x)=x\left(a^{-}\right)$, then $x\left(\left[a, a^{-}\right]\right)=$ $\left[a^{-}, a\right]$. Let $x \in$ Aut $\underline{\mathbf{M}}$ and suppose that $u(x)=x\left(a^{-}\right)$. Then $\left(\operatorname{id}_{M} \times x\right)\left(\left[a, a^{-}\right]\right) \notin U$ and $x \in U$. Since $u\left(\mathrm{id}_{M}\right)=a$, there exists $k \geqslant 1$ such that $u\left(x^{k}\right)=x^{k}\left(a^{-}\right)$ and $u\left(x^{k+1}\right)=x^{k+1}(a)$. But since $\left(x^{k}\left(a^{-}\right), x^{k+1}(a)\right) \in\left(\operatorname{id}_{M} \times x\right)\left(\left[a, a^{-}\right]\right)$, we have $\left(x^{k}\left(a^{-}\right), x^{k}(a)\right) \in\left[a, a^{-}\right]$, and so $x^{k}\left(\left[a^{-}, a\right]\right)=\left[a, a^{-}\right]$. Therefore, $x\left(\left[a, a^{-}\right]\right)=$ $\left[a^{-}, a\right]$.

Conversely, take $x \in U$ to satisfy $x\left(\left[a, a^{-}\right]\right)=\left[a^{-}, a\right]$. For every $y \in$ Aut $\underline{\mathbf{M}}$, we have that $(x \circ y)\left(\left[a, a^{-}\right]\right)=y\left(\left[a^{-}, a\right]\right)$. Hence, when $u(x \circ y) \neq x(u(y))$, either $u(y)=y\left(a^{-}\right)$, and therefore $y\left(\left[a, a^{-}\right]\right)=\left[a^{-}, a\right]$, by the first part of the proof, which implies $\left[u(y), x^{-1}(u(x \circ y))\right]=\left[y\left(a^{-}\right), y(a)\right]=\left[a, a^{-}\right]$, or $u(x \circ y)=(x \circ y)\left(a^{-}\right)$, and therefore $(x \circ y)\left(\left[a, a^{-}\right]\right)=\left[a^{-}, a\right]$, by the first part of the proof, which implies

$$
\left[u(y), x^{-1}(u(x \circ y))\right]=\left[y(a), y\left(a^{-}\right)\right]=\left[(x \circ y)\left(a^{-}\right),(x \circ y)(a)\right]=\left[a, a^{-}\right] .
$$

Then $\left(\mathrm{id}_{M} \times x\right)\left(\left[a, a^{-}\right]\right) \notin U$ and thus $u(x)=x\left(a^{-}\right)$.

Thus we may redefine the map $u$ as follows,

$$
u(x)= \begin{cases}x(a) & \text { if } x\left(\left[a, a^{-}\right]\right)=\left[a, a^{-}\right] \text {or } x \notin U ; \\ x\left(a^{-}\right) & \text {otherwise }\end{cases}
$$

and as we prove next the automorphisms in $U$ are exactly those that do not preserve $\left[a, a^{-}\right]$.

PROPOSITION 3.18. The set of minimal elements of $U$ is Aut $\underline{\mathbf{M}} \backslash H_{a, a^{-}}$. Moreover $U$ is the failset $U_{H_{a, a}}^{a a^{-}}$. 
Proof. By the previous lemma, $u(x)=x\left(a^{-}\right)$if and only if $x\left(\left[a, a^{-}\right]\right)=\left[a^{-}, a\right]$ and $x \in U$. Recall that $u(g)=g\left(a^{-}\right)$. Then $g\left(\left[a, a^{-}\right]\right)=\left[a^{-}, a\right]$. Let $x \in$ Aut $\underline{\mathbf{M}}$ and suppose that $x \notin U$. Then $u(x \circ g)=x(u(g))=(x \circ g)\left(a^{-}\right)$which implies that $(x \circ g)\left(\left[a, a^{-}\right]\right)=\left[a^{-}, a\right]$, and hence $x\left(\left[a^{-}, a\right]\right)=\left[a^{-}, a\right]$. Thus Aut $\underline{\mathbf{M}} \backslash U_{\min } \subseteq$ $H_{a, a^{-}}$. Conversely, for every $x \in H_{a, a^{-}}$and $y \in$ Aut $\underline{\mathbf{M}}$, we have that

$$
\begin{aligned}
u(y)=y\left(a^{-}\right) & \Longleftrightarrow y\left(\left[a, a^{-}\right]\right)=\left[a^{-}, a\right] \\
& \Longleftrightarrow(x \circ y)\left(\left[a, a^{-}\right]\right)=\left[a^{-}, a\right] \\
& \Longleftrightarrow u(x \circ y)=(x \circ y)\left(a^{-}\right)
\end{aligned}
$$

and so $u(x \circ y)=x(u(y))$. Hence $H_{a, a^{-}} \subseteq$ Aut $\underline{\mathbf{M}} \backslash U_{\min }$. Note that we get that $u(x)=x\left(a^{-}\right)$if and only if $x \in$ Aut $\underline{\mathbf{M}} \backslash H_{a, a^{-}}$. The rest of the proof is now immediate by applying Proposition 3.11 .

We have just proved that $U_{H_{a, a^{-}}}^{a a^{-}}$is the unique globally minimal failset $U$ that does not contain ( $\left.\operatorname{id}_{M} \times g\right)\left(\left[a, a^{-}\right]\right)$, for some $g \in U_{\min }$. By using the same arguments, we get the corresponding result for $U_{H_{a . a^{-}}}^{a^{-}}$.

Thus we have the following result.

PROPOSITION 3.19. The unique globally minimal failsets $U$ whose minimal elements are automorphisms and that do not contain one of the covers $\left(\mathrm{id}_{M} \times g\right)\left(\left[a, a^{-}\right]\right)$ and $\left(\mathrm{id}_{M} \times g\right)\left(\left[a^{-}, a\right]\right)$ of $g$, for some $g \in U_{\min }$, are $U_{H_{a, a^{-}}}^{a a^{-}}$and $U_{H_{a, a^{-}}}^{a^{-} a}$.

We still need to know if there are any other globally minimal failsets $U$ whose minimal elements are automorphisms. If so, they must contain both $\left(\mathrm{id}_{M} \times g\right)\left(\left[a, a^{-}\right]\right)$ and $\left(\mathrm{id}_{M} \times g\right)\left(\left[a^{-}, a\right]\right)$, for each one of their automorphisms $g$.

PROPOSITION 3.20. Let $U$ be a globally minimal failset whose minimal elements are automorphisms. Suppose that $\left(\mathrm{id}_{M} \times g\right)\left(\left[a, a^{-}\right]\right),\left(\operatorname{id}_{M} \times g\right)\left(\left[a^{-}, a\right]\right) \in U$, for every $g \in U_{\min }$. Then

$$
U=U_{H}^{a a^{-}}=U_{H}^{a^{-} a}=\left\{\left(\operatorname{id}_{M} \times x\right)(r) \mid x \in \text { Aut } \underline{\mathbf{M}} \backslash H \wedge \mathbf{r} \in S_{a a^{-}} \cup S_{a^{-} a}\right\},
$$

where $H=$ Aut $\underline{\mathbf{M}} \backslash U_{\min }$.

PROOF. By Propositions 3.4 and 3.6, there exist a map $u:$ End $\underline{\mathbf{M}} \rightarrow M$ and $f \in U_{\min }$ such that $U=$ Fail $_{f}(u)$ and $u(x) \in\left\{x(a), x\left(a^{-}\right)\right\}$, for every $x \in$ End $\underline{\mathbf{M}}$. By Proposition 3.11, the set $U_{H}^{a a^{-}}$is a failset of every $g \in$ Aut $\underline{\mathbf{M}} \backslash H$. We claim that $U_{H}^{a a^{-}} \subseteq U$. Let $x \in H, y \in$ Aut $\underline{\mathbf{M}} \backslash H$ and $\mathbf{r} \in S_{a a^{-}}$. By hypothesis, $\left(\right.$ id $\left._{M} \times y\right)\left(\left[a^{-}, a\right]\right) \in U$ since $y \in U_{\min }$. Then we may take $z \in$ Aut $\underline{\mathbf{M}}$ to be such that $(u(z), u(y \circ z)) \notin\left(\operatorname{id}_{M} \times y\right)\left(\left[a^{-}, a\right]\right)$, and hence $\left[u(z), y^{-1}(u(y \circ z))\right]=\left[a, a^{-}\right]$. This implies $\left(u(z), y^{-1}(u(y \circ z))\right) \notin r$ because $\left[a, a^{-}\right] \cap r=\Delta_{M}$. But now we have

$$
(u(z),(u(y \circ z))) \notin\left(\operatorname{id}_{M} \times y\right)(r)
$$


and, since $x \notin U$, we also have

$$
(u(x \circ z),(u(y \circ z)))=(x(u(z)),(u(y \circ z))) \notin(x \times y)(r) .
$$

Thus $(x \times y)(r) \in U$. Similarly we prove that $U_{H}^{a^{-} a} \subseteq U$. Then, by the minimality of $U$, we get $U_{H}^{a a^{-}}=U=U_{H}^{a^{-} a}$. Finally, observe that for every $x \in H, y \in U_{\min }$ and $r \in S_{a a^{-}} \cup S_{a^{-} a}$, we have that $(x \times y)(r)=\left(\operatorname{id}_{M} \times\left(y \circ x^{-1}\right)\right)((x \times x)(r))$, with $y \circ x^{-1} \in U_{\min }$ and $(x \times x)(r)$ also in $S_{a a^{-}} \cup S_{a^{-} a}$. Thus

$$
U=\left\{\left(\operatorname{id}_{M} \times x\right)(r) \mid x \in U_{\min } \wedge \mathbf{r} \in S_{a a^{-}} \cup S_{a^{-} a}\right\} .
$$

From the next result, it follows that every maximal proper subgroup of Aut $\underline{\mathbf{M}}$ different from $H_{a, a^{-}}$is the complement in Aut $\underline{\mathbf{M}}$ of the set of minimal elements of a unique globally minimal failset.

PkOPOSITION 3.21. Let $H$ be a maximal proper subgroup of Aut $\underline{\mathbf{M}}$ and suppose that $H \neq H_{a, a^{-}}$. Then $U_{H}^{a a^{-}}$is a globally minimal failset whose set of minimal elements is Aut $\underline{\mathbf{M}} \backslash H$. Moreover,

$$
U_{H}^{a a^{-}}=U_{H}^{a^{-a}}=\left\{\left(\operatorname{id}_{M} \times x\right)(r) \mid x \in \text { Aut } \underline{\mathbf{M}} \backslash H \wedge \mathbf{r} \in S_{a a^{-}} \cup S_{a^{-} a}\right\} .
$$

Proof. By Proposition 3.11, $U_{H}^{a a^{-}}$is a failset of every $g \in$ Aut $\underline{\mathbf{M}} \backslash H$. Let $U$ be a globally minimal failset contained in $U_{H}^{a a^{-}}$. By Proposition 3.12, the set $U_{\min }$ is Aut $\underline{\mathbf{M}} \backslash H$. Since $H \neq H_{a, a^{-}}$, and therefore $U \neq U_{H_{a, a^{-}}}^{a a^{-}}, U_{H_{a, a^{-}}}^{a^{-}}$, we must have that $\left(\mathrm{id}_{M} \times g\right)\left(\left[a, a^{-}\right]\right),\left(\operatorname{id}_{M} \times g\right)\left(\left[a^{-}, a\right]\right) \in U$, for every $g \in U_{\min }$, by applying Proposition 3.19. Finally

$$
U=U_{H}^{a a^{-}}=U_{H}^{a^{-a}}=\left\{\left(\mathrm{id}_{M} \times x\right)(r) \mid x \in U_{\min } \wedge \mathbf{r} \in S_{a a^{-}} \cup S_{a^{-} a}\right\},
$$

by Proposition 3.20.

COROLlaRY 3.22. Let $H$ be a proper subgroup of Aut $\underline{\mathbf{M}}$ and suppose that $H \neq$ $H_{a, a^{-}}$. If $U=\left\{\left(\mathrm{id}_{M} \times x\right)(r) \mid x \in\right.$ Aut $\left.\underline{\mathbf{M}} \backslash H \wedge \mathbf{r} \in S_{a a^{-}} \cup S_{a^{-} a}\right\}$ is a globally minimal failset, then $H$ is a maximal proper subgroup of Aut $\underline{\mathbf{M}}$.

Proof. Take $H^{\prime}$ to be a maximal proper subgroup of Aut $\underline{\mathrm{M}}$ such that $H \subseteq H^{\prime}$. It follows from the last proposition that

$$
U_{H^{\prime}}^{a a^{-}}=\left\{\left(\operatorname{id}_{M} \times x\right)(r) \mid x \in \operatorname{Aut} \underline{\mathbf{M}} \backslash H^{\prime} \wedge \mathbf{r} \in S_{a a^{-}} \cup S_{a^{-} a}\right\}
$$

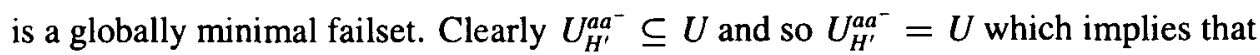
Aut $\underline{\mathbf{M}} \backslash H^{\prime}=$ Aut $\underline{\mathbf{M}} \backslash H$. 
Now we combine the last corollary and the previous three propositions and we obtain the theorem below.

THEOREM 3.23. Let $\left[a, a^{-}\right]$and $\left[a^{-}, a\right]$ be the unique two covers of $\Delta_{M}$ in $\$\left(\underline{M}^{2}\right)$ and suppose that $x\left(\left[a, a^{-}\right]\right)=\left[a^{-}, a\right]$, for some $x \in$ Aut $\underline{\mathbf{M}}$. The globally minimal failsets whose minimal elements are automorphisms are exactly the following sets:

(a) $\quad U_{H_{a, a^{-}}}^{a a^{-}}=\left\{\left(\left(\mathrm{id}_{M} \times g\right)(r),\left(\left(\mathrm{id}_{M} \times g\right)(r)\right)^{\smile} \mid g \in\right.\right.$ Aut $\left.\underline{\mathbf{M}} \backslash H_{a, a^{-}} \wedge \mathbf{r} \in S_{a a^{-}}\right\}$;

(b) $U_{H_{a, a^{-}}}^{a^{-a}}=\left\{\left(\mathrm{id}_{M} \times g\right)(r),\left(\left(\mathrm{id}_{M} \times g\right)(r)\right)^{\smile} \mid g \in\right.$ Aut $\left.\underline{\mathbf{M}} \backslash H_{a, a^{-}} \wedge \mathbf{r} \in S_{a^{-a} a}\right\}$;

(c) $U_{H}^{a a^{-}}=U_{H}^{a^{-a}}=\left\{\left(\mathrm{id}_{M} \times g\right)(r) \mid g \in\right.$ Aut $\left.\underline{\mathbf{M}} \backslash H \wedge \mathbf{r} \in S_{a a^{-}} \cup S_{a^{-} a}\right\}$, where $H$ is $a$ maximal proper subgroup of Aut $\underline{\mathbf{M}}$, distinct from $H_{a, a^{2}}$.

When the globally minimal failsets $U$ that satisfy $U_{\min } \subseteq$ Aut $\underline{\mathbf{M}}$ are determined by the maximal proper subgroups $H$ of Aut $\underline{\mathbf{M}}$ in a way that $U_{\min }=$ Aut $\underline{\mathbf{M}} \backslash H$, a given set of automorphisms of $\underline{\mathbf{M}}$ is a transversal of the family of globally minimal failsets, whose minimal elements are automorphisms of $\underline{\mathbf{M}}$, if and only if it is a minimal generating set of Aut $\underline{\mathbf{M}}$. Thus, the subdirect irreducibility of $\underline{\mathbf{M}}$ allows us to take any minimal generating set of Aut $\underline{\mathbf{M}}$ as a transversal of the globally minimal failsets we have been describing here.

\section{Aut-free globally minimal failsets}

In the preceding section we considered the globally minimal failsets whose minimal elements are automorphisms. Their description was given in case the generating algebra $\underline{\mathbf{M}}$ of the quasivariety $\mathscr{A}$ is a finite subdirectly irreducible algebra with a definable lattice-structure. Now we aim to describe the globally minimal failsets whose minimal elements are either endomorphisms of $\underline{\mathbf{M}}$ or the converses of their graphs. We first prove that such a failset cannot intersect Aut $\underline{\mathbf{M}}$ if it contains an endomorphism in End $\underline{\mathbf{M}} \backslash$ Aut $\underline{\mathbf{M}}$. Therefore, here we only consider aut-free globally minimal failsets, which we define to be globally minimal failsets whose minimal elements belong to the set

$$
\left\{\text { graph } f,(\operatorname{graph} f)^{\smile} \mid f \in \operatorname{End} \underline{\mathbf{M}} \backslash \text { Aut } \underline{\mathbf{M}}\right\} .
$$

Throughout this section we assume that the quasivariety $\mathscr{A}=\mathbb{D S P}(\underline{\mathbf{M}})$ is generated by a finite lattice-structured algebra $\underline{\mathbf{M}}$.

PROPOSITION 4.1. Let $U$ be a globally minimal failset whose minimal elements are either endomorphisms of $\underline{\mathbf{M}}$ or converses of graphs of endomorphisms of $\underline{\mathbf{M}}$. If the algebra $\underline{\mathbf{M}}$ is subdirectly irreducible, then either $U$ contains no automorphisms of $\underline{\mathbf{M}}$ or every minimal element of $U$ is an automorphism of $\underline{\mathbf{M}}$. 
Proof. Suppose that $U \cap$ Aut $\underline{\mathbf{M}} \neq \emptyset$. Then take $g \in U \cap$ Aut $\underline{\mathbf{M}}$. By [9, Proposition 3.1.1], there exists a map $u:$ End $\underline{\mathbf{M}} \rightarrow M$ such that $U=$ Fail $_{g}(u)$ and $u(g)=g(c), u\left(\operatorname{id}_{M}\right)=d$, with $c \neq d$. There exists $a \in \mathscr{J}(\underline{\mathbf{M}})$ such that either $a \leqslant c$ and $a \notin d$, or $a \notin c$ and $a \leqslant d$. Now take $a^{\prime}, b^{\prime} \in M$ such that $\left[a^{\prime}, b^{\prime}\right] \succ_{M}$ and $\left[a^{\prime}, b^{\prime}\right] \subseteq\left[a, a^{-}\right]$. Define a map $v:$ End $\underline{\mathbf{M}} \rightarrow M$ by

$$
v(x)= \begin{cases}x\left(a^{\prime}\right) & \text { if } x(a) \leqslant u(x) ; \\ x\left(b^{\prime}\right) & \text { otherwise. }\end{cases}
$$

For every $f \in \operatorname{End} \underline{\mathbf{M}} \backslash$ Aut $\underline{\mathbf{M}}$, we have that $\theta\left(a^{\prime}, b^{\prime}\right) \subseteq \operatorname{ker} f$ because $\theta\left(a^{\prime}, b^{\prime}\right)$ is the atom of Con $\underline{\mathbf{M}}$ (see [6, Proposition 1.8]). Then, for every $x \in \operatorname{End} \underline{\mathbf{M}}$, we have $(f \circ x)\left(a^{\prime}\right)=(f \circ x)\left(b^{\prime}\right)$ which implies that $v(f \circ x)=f(v(x))$. Consequently, Fail $_{g}(v)$ does not intersect End $\underline{\mathbf{M}} \backslash$ Aut $\underline{\mathbf{M}}$ and also $\left(f_{1} \cap f_{2}\right):=\left\{\left(f_{1}(x), f_{2}(x)\right) \mid\right.$ $x \in M\} \notin U$, for every $f_{1}, f_{2} \in$ End $\underline{\mathbf{M}} \backslash$ Aut $\underline{\mathbf{M}}$. We claim that $U=$ Fail $_{g}(v)$, and therefore every minimal element of $U$ is an automorphism of $\underline{\mathbf{M}}$. Observe that either $g(a) \leqslant g(c)=u(g)$ and $a \notin d=u\left(\mathrm{id}_{M}\right)$, or $a \leqslant d=u\left(\mathrm{id}_{M}\right)$ and $g(a) \nless g(c)=u(g)$. Therefore, $v(g) \neq g\left(v\left(\mathrm{id}_{M}\right)\right)$ and so Fail $g(v)$ is a failset of $g$. Now, by the minimality of $U$ as a failset of $g$, we only need to prove that Fail $_{g}(v) \subseteq U$. Take $s \in$ Fail $_{g}(v)$. There exist $x, y \in$ End $\underline{M}$ such that $(x, y) \in s$ and $(v(x), v(y)) \notin s$. We may assume that $(v(x), v(y))=\left(x\left(a^{\prime}\right), y\left(b^{\prime}\right)\right)$. Then $x(a) \leqslant u(x)$ and $y(a) \notin u(y)$. Note that $\left(a^{\prime}, b^{\prime}\right) \notin$ ker $y$ (otherwise $(v(x), v(y))=$ $\left.\left(x\left(a^{\prime}\right), y\left(a^{\prime}\right)\right) \in s\right)$. Thus $y \in$ Aut $\underline{\mathbf{M}}$, and so $y\left(a^{-}\right) \prec y(a)$ and $y(a) \in \mathscr{J}(\underline{\mathbf{M}})$ (see [6, Proposition 1.1]). Consequently, $y\left(a^{-}\right)=(y(a) \wedge u(y)) \vee y\left(a^{-}\right)$and we have that

$$
\begin{aligned}
\left(x(a), y\left(a^{-}\right)\right) & =\left((x(a) \wedge u(x)) \vee x\left(a^{-}\right),(y(a) \wedge u(y)) \vee y\left(a^{-}\right)\right) \\
& =((x(a), y(a)) \wedge(u(x), u(y))) \vee\left(x\left(a^{-}\right), y\left(a^{-}\right)\right),
\end{aligned}
$$

and $\left(x(a), y\left(a^{-}\right)\right) \notin s$, or otherwise $\left(x\left(a^{\prime}\right), y\left(b^{\prime}\right)\right) \in(x \times y)\left(\left[a, a^{-}\right]\right) \subseteq s$. Then we must have $(u(x), u(y)) \notin s$ since $(x(a), y(a)),\left(x\left(a^{-}\right), y\left(a^{-}\right)\right) \in s$. Thus $s \in U$.

Note that the binary relation $\theta$ defined on End $\underline{\mathbf{M}}$ by $x \theta y$ if and only if $x \in($ Aut $\underline{\mathbf{M}}) y$ is an equivalence relation. Hence take $f_{0}, \ldots, f_{n} \in$ End $\underline{\mathbf{M}}$ to be such that $f_{0}=\mathrm{id}_{M}$ and $f_{0} /{ }_{\theta}, \ldots, f_{n} /{ }_{\theta}$ are the equivalence classes modulo $\theta$.

Let $U$ be an aut-free globally minimal failset. Then $U \cap$ Aut $\underline{\mathbf{M}}=\emptyset$ and thereby, for each $i \in(1, \ldots, n)$, we have $f_{i} \in U$ if and only if (Aut $\underline{\mathbf{M}}$ ) $f_{i}$ is a subset of $U_{\min }$. Also observe that every map $u:$ End $\underline{\mathbf{M}} \rightarrow M$ that satisfies $U=\operatorname{Fail}_{f}(u)$, for some $f \in$ End $\underline{\mathbf{M}}$, is completely determined by the values that it takes on each endomorphism $f_{i}$, with $i \in\{0, \ldots, n\}$; moreover $u\left(f_{i}\right) \in f_{i}(M)$ for every $i \in$ $\{0, \ldots, n\}$ since $\operatorname{id}_{f_{i}(M)} \notin U$.

Whence take $f \in U_{\min }$ and take $a_{i} \in M$, with $i \in\{0, \ldots, n\}$ to satisfy $U=$ Fail $_{f}(u)$, where $u$ : End $\underline{\mathbf{M}} \rightarrow M$ is defined by $u\left(x \circ f_{i}\right)=\left(x \circ f_{i}\right)\left(a_{i}\right)$, for each 
$x \in$ Aut $\underline{\mathrm{M}}$ and each $i \in\{0, \ldots, n\}$. For $i, j \in(1, \ldots, n)$, note that

$$
\rho_{1}\left(\left(f_{i} \sqcap f_{j}\right)(M)\right)=f_{i}(M) \subsetneq M \quad \text { and } \quad \rho_{2}\left(\left(f_{i} \sqcap f_{j}\right)(M)\right)=f_{j}(M) \subsetneq M .
$$

Hence $\left(f_{i} \sqcap f_{j}\right)(M) \notin U$ because every $r \in U_{\text {min }}$ satisfies $\rho_{1}(r)=M$ or $\rho_{2}(r)=M$. Then $f \in U$ must be witnessed by $(x, f \circ x)$, for some $x \in$ Aut $\underline{\mathbf{M}}$. Let $i \in(1, \ldots, n)$ and $y \in$ Aut $\underline{\mathbf{M}}$ such that $f \circ x=y \circ f_{i}$. It follows that

$$
\left(y \circ f_{i}\right)\left(a_{i}\right)=u(f \circ x) \neq f(u(x))=f\left(x\left(a_{0}\right)\right)=\left(y \circ f_{i}\right)\left(a_{0}\right)
$$

and so $\left[a_{0}, a_{i}\right]_{f_{i}}>\operatorname{ker} f_{i}$, where $\left[a_{0}, a_{i}\right]_{f_{i}}$ denotes the subalgebra of $\underline{\mathbf{M}}^{2}$ generated by $\operatorname{ker} f_{i} \cup\left\{\left(a_{0}, a_{i}\right)\right\}$.

The following result allows us to assume that $\left[a_{0}, a_{i}\right]_{f_{i}} \succ \operatorname{ker} f_{i}$, for every $i \in$ $(1, \ldots, n)$ such that $\left[a_{0}, a_{i}\right]_{f_{i}}>\operatorname{ker} f_{i}$.

Proposition 4.2. There exist $a_{0}^{\prime}, \ldots, a_{n}^{\prime} \in M$ for which the following conditions hold:

(a) For every $i \in(1, \ldots, n)$, if $\left(a_{0}^{\prime}, a_{i}^{\prime}\right) \notin \operatorname{ker} f_{i}$ then $\left[a_{0}^{\prime}, a_{i}^{\prime}\right]_{f_{i}} \succ \operatorname{ker} f_{i}$.

(b) $U=$ Fail $_{f}(v)$, where $v$ : End $\underline{\mathbf{M}} \rightarrow M$ is the map defined by

$$
\forall i \in\{0, \ldots, n\} \forall x \in(\text { Aut } \underline{\mathbf{M}}) f_{i} \quad\left(v(x)=x\left(a_{i}^{\prime}\right)\right) .
$$

Proof. Let $i_{0}$ be the least $i \in(1, \ldots, n)$ such that $f_{i}\left(a_{i}\right) \neq f_{i}\left(a_{0}\right)$ but $\left[a_{0}, a_{i}\right]_{f_{i}}$ does not cover $\operatorname{ker} f_{i}$ in $\mathbb{S}\left(\underline{\mathbf{M}}^{2}\right)$. Let $c, d \in M$ satisfy $[c, d]_{f_{b}} \succ \operatorname{ker} f_{i_{0}}$ and $[c, d]_{f_{b}} \subseteq$ $\left[a_{0}, a_{i_{0}}\right]_{f_{i_{0}}}$. There exists an $\mathbf{m}$-ary term $\boldsymbol{t}$ of the same type as that of $\underline{\mathbf{M}}$ such that

$$
(c, d)=t^{M^{2}}\left(\left(c_{1}, d_{1}\right), \ldots,\left(c_{m}, d_{m}\right)\right)=\left(t^{M}\left(c_{1}, \ldots, c_{m}\right), t^{M}\left(d_{1}, \ldots, d_{m}\right)\right),
$$

where either $\left(c_{k}, d_{k}\right) \in \operatorname{ker} f_{i_{0}}$ or $\left(c_{k}, d_{k}\right)=\left(a_{0}, a_{i_{0}}\right)$, for $k \in(1, \ldots, m)$. For every $i \in\{0, \ldots, n\}$, take $a_{i}^{\prime}=t^{M}\left(c_{1}^{i}, \ldots, c_{m}^{i}\right)$, where

$$
c_{k}^{i}= \begin{cases}c_{k} & \text { if }\left(c_{k}, d_{k}\right) \in \operatorname{ker} f_{i_{0}} ; \\ a_{i} & \text { otherwise, }\end{cases}
$$

for $k \in(1, \ldots, m)$. For $i \in(1, \ldots, n)$, observe that $\left(a_{0}^{\prime}, a_{i}^{\prime}\right) \in\left[a_{0}, a_{i}\right]_{f_{i}}$ and hence either $\left(a_{0}^{\prime}, a_{i}^{\prime}\right) \in \operatorname{ker} f_{i}$ or $\left[a_{0}^{\prime}, a_{i}^{\prime}\right]_{f_{i}}>\operatorname{ker} f_{i}$ whenever $\left[a_{0}, a_{i}\right]_{f_{i}}>\operatorname{ker} f_{i}$. Now define a map $v:$ End $\underline{\mathbf{M}} \rightarrow M$ by

$$
\forall i \in\{0, \ldots, n\} \forall x \in(\text { Aut } \underline{\mathrm{M}}) f_{i} \quad\left(v(x)=x\left(a_{i}^{\prime}\right)\right) .
$$

Note that $a_{0}^{\prime}=c$ and $f_{i_{0}}\left(a_{i_{0}}^{\prime}\right)=t^{M}\left(f_{i_{0}}\left(c_{1}^{i_{0}}\right), \ldots, f_{i_{0}}\left(c_{m}^{i_{0}}\right)\right)=f_{i_{0}}(d)$ since either $c_{k}^{i_{0}}=$ $a_{i_{0}}=d_{k}$ or $f_{i_{0}}\left(c_{k}^{i_{0}}\right)=f_{i_{0}}\left(c_{k}\right)=f_{i_{0}}\left(d_{k}\right)$, for $k \in(1, \ldots, m)$. So

$$
v\left(f_{i_{0}}\right)=f_{i_{0}}(d) \neq f_{i_{0}}(c)=f_{i_{0}}\left(v\left(\mathrm{id}_{M}\right)\right) \text {. }
$$


Therefore, Fail $f_{f_{0}}(v)$ is a failset of $f_{i_{0}}$. We claim that Fail $f_{f_{0}}(v) \subseteq U$. Take $s \in$ Fail $_{f_{i_{0}}}(v)$. There exist $x, y \in$ End $\underline{\mathbf{M}}$ such that $(x, y) \in s$ and $(v(x), v(y)) \notin s$. Let $i, j \in\{0, \ldots, n\}$ such that $x \in($ Aut $\underline{\mathbf{M}}) f_{i}$ and $y \in($ Aut $\underline{\mathbf{M}}) f_{j}$. Then

$$
\begin{aligned}
(v(x), v(y)) & =\left(x\left(t^{M}\left(c_{1}^{i}, \ldots, c_{m}^{i}\right)\right), y\left(t^{M}\left(c_{1}^{j}, \ldots, c_{m}^{j}\right)\right)\right) \\
& =t^{M^{2}}\left(\left(x\left(c_{1}^{i}\right), y\left(c_{1}^{j}\right)\right), \ldots,\left(x\left(c_{m}^{i}\right), y\left(c_{m}^{j}\right)\right)\right)
\end{aligned}
$$

and for each $k \in(1, \ldots, m)$, we have that either

$$
\begin{aligned}
& \left(x\left(c_{k}^{i}\right), y\left(c_{k}^{j}\right)\right)=\left(x\left(c_{k}\right), y\left(c_{k}\right)\right) \in s \text { or } \\
& \left(x\left(c_{k}^{i}\right), y\left(c_{k}^{j}\right)\right)=\left(x\left(a_{i}\right), y\left(a_{j}\right)\right)=(u(x), u(y)) .
\end{aligned}
$$

Then $(v(x), v(y)) \notin s$ implies that $(u(x), u(y)) \notin s$, and so $s \in U$. Now recall that $U$ is a minimal failset of $f_{i_{0}}$ and consequently $U=$ Fail $_{f_{i_{0}}}(v)$.

If there is still some $i \in(1, \ldots, n)$ such that $f_{i}\left(a_{i}^{\prime}\right) \neq f_{i}\left(a_{0}^{\prime}\right)$ and $\left[a_{0}^{\prime}, a_{i}^{\prime}\right]_{f_{i}}$ does not cover $\operatorname{ker} f_{i}$, then we repeat the procedure until we get that $\left(a_{0}^{\prime}, a_{i}^{\prime}\right) \in \operatorname{ker} f_{i}$ or $\left[a_{0}^{\prime}, a_{i}^{\prime}\right]_{f_{i}} \succ \operatorname{ker} f_{i}$, for every $i \in(1, \ldots, n)$. Then we get elements $a_{0}^{\prime}, \ldots, a_{n}^{\prime}$ in $M$ and a map $v:$ End $\underline{\mathbf{M}} \rightarrow M$ under the conditions required.

Hence we may assume that $f=f_{1}$ and $u(f) \neq f\left(u\left(\operatorname{id}_{M}\right)\right)$. Therefore, $\left[a_{0}, a_{1}\right]_{f} \succ$ $\operatorname{ker} f$.

Since $f\left(a_{0}\right) \neq f\left(a_{1}\right)$, there is $a \in \mathscr{J}(\underline{\mathbf{M}})$ such that $\left(a, a^{-}\right) \notin \operatorname{ker} f$ and

$$
\begin{gathered}
\left(\left(f\left(a_{0}\right), f\left(a_{1}\right)\right) \wedge(f(a), f(a))\right) \vee\left(f\left(a^{-}\right), f\left(a^{-}\right)\right) \\
\in\left\{\left(f(a), f\left(a^{-}\right)\right),\left(f\left(a^{-}\right), f(a)\right)\right\}
\end{gathered}
$$

(see [6, Lemma 1.5 and Proposition 1.3]). Thus we have

$$
\left(f\left(\left(a_{0} \wedge a\right) \vee a^{-}\right), f\left(\left(a_{1} \wedge a\right) \vee a^{-}\right)\right) \in\left\{\left(f(a), f\left(a^{-}\right)\right),\left(f\left(a^{-}\right), f(a)\right)\right\}
$$

and so $\left(\left(\left(a_{0} \wedge a\right) \vee a^{-}\right),\left(a_{1} \wedge a\right) \vee a^{-}\right) \notin \operatorname{ker} f$. Since

$$
\left(\left(a_{0} \wedge a\right) \vee a^{-},\left(a_{1} \wedge a\right) \vee a^{-}\right)=\left(\left(a_{0}, a_{1}\right) \wedge(a, a)\right) \vee\left(a^{-}, a^{-}\right) \in\left[a_{0}, a_{1}\right]_{f}
$$

we have $\left[\left(a_{0} \wedge a\right) \vee a^{-},\left(a_{1} \wedge a\right) \vee a^{-}\right]_{f}=\left[a_{0}, a_{1}\right]_{f} \succ \operatorname{ker} f$. Finally, observe that $\left(\left(a_{0} \wedge a\right) \vee a^{-},\left(a_{1} \wedge a\right) \vee a^{-}\right)$is either $\left(a, a^{-}\right)$or $\left(a^{-}, a\right)$.

Now define a map $v:$ End $\underline{\mathbf{M}} \rightarrow M$ by

$$
\forall i \in\{0, \ldots, n\} \forall x \in(\text { Aut } \underline{\mathbf{M}}) f_{i}\left(v(x)=x\left(\left(a_{i} \wedge a\right) \vee a^{-}\right)\right) .
$$

Observe that $v(y) \in\left\{y(a), y\left(a^{-}\right)\right\}$, for all $y \in$ End $\underline{\mathbf{M}}$, because $a \in \mathscr{J}(\underline{\mathbf{M}})$ and $a>a^{-}$. 
LEMMA 4.3. The globally minimal failset $U$ is Fail $_{f}(v)$.

Proof. Note that $\left(v(f), f\left(v\left(\operatorname{id}_{M}\right)\right)\right.$ is either $\left(f(a), f\left(a^{-}\right)\right)$or $\left(f\left(a^{-}\right), f(a)\right)$, and so $v(f) \neq f\left(v\left(\mathrm{id}_{M}\right)\right)$. Hence Fail $_{f}(v)$ is a failset of $f$. By the minimality of $U$ as a failset of $f$, we only need to show that $\operatorname{Fail}_{f}(v) \subseteq U$. Take $s \in$ Fail $_{f}(v)$. There exist $x, y \in$ End $\underline{\mathbf{M}}$ such that $(x, y) \in s$ and $(v(x), v(y)) \notin s$. Let $i, j \in\{0, \ldots, n\}$ such that $x \in($ Aut $\underline{\mathbf{M}}) f_{i}$ and $y \in($ Aut $\underline{\mathbf{M}}) f_{j}$. Note that

$$
(v(x), v(y))=\left(\left(x\left(a_{i}\right), y\left(a_{j}\right)\right) \wedge(x(a), y(a))\right) \vee\left(x\left(a^{-}\right), y\left(a^{-}\right)\right)
$$

and $(x(a), y(a)),\left(x\left(a^{-}\right), y\left(a^{-}\right)\right) \in s$. Consequently $(u(x), u(y))=\left(x\left(a_{i}\right), y\left(a_{j}\right)\right) \notin$ $s$. Thus $s \in U$.

Let $x \in$ End $\underline{M}$ and suppose that $v(x) \neq x\left(v\left(\operatorname{id}_{M}\right)\right)$. Observe that $\left[a, a^{-}\right]_{x}$ covers $\operatorname{ker} x$ :

There exists $i \in(1, \ldots, n)$ such that $x \in($ Aut $\underline{\mathbf{M}}) f_{i}$. Note that $\operatorname{ker} x=\operatorname{ker} f_{i}$ and so $\left[a, a^{-}\right]_{x}=\left[a, a^{-}\right]_{f_{i}}$. If $v(x) \neq x\left(v\left(\operatorname{id}_{M}\right)\right)$, that is, $x\left(\left(a_{i} \wedge a\right) \vee a^{-}\right) \neq$ $x\left(\left(a_{0} \wedge a\right) \vee a^{-}\right)$, then $x\left(a_{i}\right) \neq x\left(a_{0}\right)$ and so $\left(a_{0}, a_{i}\right) \notin \operatorname{ker} f_{i}$ which implies that $\left[a_{0}, a_{i}\right]_{f_{i}} \succ \operatorname{ker} f_{i}$. Finally, observe that $\left(\left(a_{0} \wedge a\right) \vee a^{-},\left(a_{i} \wedge a\right) \vee a^{-}\right) \in\left[a_{0}, a_{i}\right]_{f_{i}} \backslash \operatorname{ker} f_{i}$ and $\left(\left(a_{0} \wedge a\right) \vee a^{-},\left(a_{i} \wedge a\right) \vee a^{-}\right)$is either $\left(a, a^{-}\right)$or $\left(a^{-}, a\right)$. Then $\left[a, a^{-}\right]_{f_{i}}$ is either $\left[a_{0}, a_{i}\right]_{f_{i}}$ or $\left[a_{i}, a_{0}\right]_{f_{i}}$. Therefore, $\left[a, a^{-}\right]_{f_{i}} \succ \operatorname{ker} f_{i}$.

\section{LEMMA 4.4. The following condition holds}

$$
\forall x \in \text { End } \underline{\mathbf{M}} \backslash \text { Aut } \underline{\mathbf{M}} v\left(\operatorname{id}_{M}\right)=a \Longleftrightarrow v(x)=x\left(a^{-}\right) .
$$

PROOF. Recall that $\left(f\left(v\left(\operatorname{id}_{M}\right)\right), v(f)\right) \in\left\{\left(f(a), f\left(a^{-}\right)\right),\left(f\left(a^{-}\right), f(a)\right)\right\}$ and $f(a) \neq f\left(a^{-}\right)$. Hence, if $v(x)=x\left(a^{-}\right)$, for every $x \in$ End $\underline{\mathbf{M}} \backslash$ Aut $\underline{\mathbf{M}}$, and in particular $v(f)=f\left(a^{-}\right)$, we must have $v\left(\mathrm{id}_{M}\right)=a$. Now suppose that $v\left(\mathrm{id}_{M}\right)=a$ and let $x \in$ End $\underline{\mathbf{M}} \backslash$ Aut $\underline{\mathbf{M}}$. If $v(x)=x(a) \neq x\left(a^{-}\right)$, then there exists $c \in M$ such that $x(a)=x(c)$ and $f\left(a^{-}\right)=f(c)$ because $\left(x(a), f\left(a^{-}\right)\right)=(v(x), v(f)) \in(x \sqcap f)(M)$ (recall that $x, f \notin$ Aut $\underline{\mathbf{M}}$ implies that $(x \sqcap f)(M) \notin U$ ). But then we have that

$$
x\left((a \wedge c) \vee a^{-}\right)=(x(a) \wedge x(c)) \vee x\left(a^{-}\right)=x(a) \vee x\left(a^{-}\right)=x(a) \neq x\left(a^{-}\right)
$$

and this implies that $a \leqslant c$. Consequently,

$$
f(a)=f(a \wedge c)=f(a) \wedge f(c)=f(a) \wedge f\left(a^{-}\right)=f\left(a^{-}\right)
$$

and we get a contradiction.

Now the proposition below follows immediately. 
PROPOSITION 4.5. Let $U$ be an aut-free globally minimal failset. Then $U=$ Fail $_{f}(u)$, for some $f \in$ End $\underline{\mathbf{M}} \backslash$ Aut $\underline{\mathbf{M}}$ where $u:$ End $\underline{\mathbf{M}} \rightarrow M$ is the map defined by

$$
u(x)= \begin{cases}x(a) & \text { if } x \in \text { Aut } \underline{\mathbf{M}} \\ x(b) & \text { otherwise }\end{cases}
$$

for some $a, b \in M$ that satisfy the following conditions:

(a) Either $a \in \mathscr{J}(\underline{\mathbf{M}})$ and $b=a^{-}$, or $b \in \mathscr{J}(\underline{\mathbf{M}})$ and $a=b^{-}$.

(b) For every $x \in$ End $\underline{\mathbf{M}} \backslash$ Aut $\underline{\mathbf{M}}$ if $(a, b) \notin \operatorname{ker} x$ then $[a, b]_{x}>\operatorname{ker} x$.

Let $a \in \mathscr{J}(\underline{\mathbf{M}})$ and let $b=a^{-}$. The following are equivalent:

(i) $(a, b) \notin \operatorname{ker} f$, for some $f \in \operatorname{End} \underline{\mathbf{M}} \backslash$ Aut $\underline{\mathbf{M}}$, and $[a, b]_{x} \succ \operatorname{ker} x$ whenever $x \in$ End $\underline{\mathbf{M}} \backslash$ Aut $\underline{\mathbf{M}}$ and $(a, b) \notin \operatorname{ker} x$;

(ii) $(a, b) \notin \kappa$ and $\operatorname{ker} x \vee \theta(a, b) \succ \operatorname{ker} x$ in $\operatorname{Con} \underline{\mathbf{M}}$ whenever $x \in$ End $\underline{\mathbf{M}} \backslash$ Aut $\underline{\mathbf{M}}$ and $(a, b) \notin \operatorname{ker} x$;

(iii) $\kappa \vee \theta(a, b) \succ \kappa$ in $\operatorname{Con} \underline{\mathbf{M}}$;

where $\kappa=\bigcap(\operatorname{ker} f \mid f \in$ End $\underline{\mathbf{M}} \backslash$ Aut $\underline{\mathbf{M}}$ ) as defined in Section 3 (see [6, Propositions 1.8 and 1.16]).

Hence, we may reformulate Proposition 4.5 by replacing condition (b) by the condition below,

$$
\kappa \vee \theta(a, b) \text { is a cover of } \kappa \text { in } \operatorname{Con} \underline{\mathbf{M}} \text {. }
$$

At this stage we already know where to search for maps $u$ that define aut-free globally minimal failsets. Next we are going to describe the elements of these failsets.

Denote by $\mathscr{J}_{\kappa}$ the set of all non-zero join-irreducible elements $a$ for which $\kappa \vee$ $\theta\left(a, a^{-}\right)$is a cover of $\kappa$ in the congruence lattice Con $\underline{\mathbf{M}}$.

For every $a \in \mathscr{J}_{k}$, we denote by

$I_{a} \quad$ the set $\left\{i \in\{1, \ldots, n\} \mid\left(a, a^{-}\right) \notin \operatorname{ker} f_{i}\right\}$;

$S_{a a^{-}}^{i}$ the set $\left\{\mathbf{r} \in \mathbb{S}\left(\underline{\mathbf{M}}^{2}\right) \mid\left(\operatorname{id}_{M} \times f_{i}\right)^{-1}(\mathbf{r}) \cap\left[a, a^{-}\right]_{f_{i}}=\operatorname{ker} f_{i}\right\}$;

$S_{a^{-} a}^{i}$ the set $\left\{\mathbf{r} \in \mathbb{S}\left(\underline{\mathbf{M}}^{2}\right) \mid\left(\operatorname{id}_{M} \times f_{i}\right)^{-1}(\mathbf{r}) \cap\left[a^{-}, a\right]_{f_{i}}=\operatorname{ker} f_{i}\right\}$.

For $a \in \mathscr{J}_{\kappa}, i \in I_{a}$ and $\mathbf{r} \leqslant \underline{\mathbf{M}}^{2}$, observe that

$$
\begin{aligned}
\left(\mathrm{id}_{M}, f_{i}\right) & \in r \wedge\left(c, f_{i}(d)\right) \notin r \\
& \Longleftrightarrow \operatorname{ker} f_{i} \subseteq\left(\mathrm{id}_{M} \times f_{i}\right)^{-1}(r) \wedge(c, d) \notin\left(\mathrm{id}_{M} \times f_{i}\right)^{-1}(r) \\
& \Longleftrightarrow\left(\operatorname{id}_{M} \times f_{i}\right)^{-1}(r) \cap[c, d]_{f_{i}}=\operatorname{ker} f_{i} \Longleftrightarrow \mathbf{r} \in S_{c d}^{i},
\end{aligned}
$$

where $c, d \in M$ and $\{c, d\}=\left\{a, a^{-}\right\}$. Thus, $S_{a a^{-}}^{i}$ is the set of subalgebras $\mathbf{r}$ of $\underline{\mathbf{M}}^{2}$ that contain the graph of $f_{i}$ but do not contain $\left(a, f_{i}\left(a^{-}\right)\right)$, and $S_{a^{-} a}^{i}$ is the set of subalgebras $\mathbf{r}$ of $\underline{\mathbf{M}}^{2}$ that contain the graph of $f_{i}$ but do not contain $\left(a^{-}, f_{i}(a)\right)$. 
PROPOSITION 4.6. Let $U$ be an aut-free globally minimal failset. Then the minimal elements of $U$ are the endomorphisms of $\underline{\mathbf{M}}$ that belong to the set

$$
\bigcup\left\{(\text { Aut } \underline{\mathbf{M}}) f_{i}(\text { Aut } \underline{\mathbf{M}}) \mid i \in I_{a}\right\}
$$

for some $a \in \mathscr{J}_{k}$, and the converses of their graphs.

Proof. Take $a$ and $b$ to be elements of $M$ under the conditions of Proposition 4.5. Recall that $U_{\min } \subseteq$ End $\underline{\mathbf{M}} \backslash$ Aut $\underline{\mathbf{M}}$. Let $x \in$ End $\underline{\mathbf{M}} \backslash$ Aut $\underline{\mathbf{M}}$. Then $x \in U$ if and only if there exists $y \in$ Aut $\underline{\mathbf{M}}$ such that $u(x \circ y) \neq x(u(y))$, that is, $(a, b) \notin \operatorname{ker} x \circ y$. Hence, $x \in U$ if and only if there exist $y, z \in$ Aut $\underline{\mathbf{M}}$ and $i \in I_{a}$ such that $x \circ y=z \circ f_{i}$ or equivalently there exists $i \in I_{a}$ such that $x \in($ Aut $\underline{\mathbf{M}}) f_{i}$ (Aut $\underline{\mathbf{M}}$ ).

For every $c, d \in M$, let $u_{c d}:$ End $\underline{\mathbf{M}} \rightarrow M$ be the map defined by

$$
u_{c d}(x)= \begin{cases}x(c) & \text { if } x \in \text { Aut } \underline{\mathbf{M}} \\ x(d) & \text { otherwise }\end{cases}
$$

LEMMA 4.7. Let $a \in \mathscr{J}_{\kappa}$. If $U=$ Fail $_{f}\left(u_{c d}\right)$, for some $f \in$ End $\underline{\mathbf{M}}$ and $c, d \in M$ such that $\{c, d\}=\left\{a, a^{-}\right\}$, then

$$
U=\bigcup_{i \in l_{a}}\left\{(x \times y)(r),((x \times y)(r))^{\smile} \mid x, y \in \operatorname{Aut} \underline{\mathbf{M}} \wedge \mathbf{r} \in S_{c d}^{i}\right\}
$$

Proof. For every $s \subseteq M^{2}$, we have that $s \in U$ if and only if there are $x, y \in$ Aut $\underline{\mathbf{M}}$ and $i \in I_{a}$ such that

$$
\begin{aligned}
& \text { either }\left(x, y \circ f_{i}\right) \in s \quad \text { and } \quad\left(x(c),\left(y \circ f_{i}\right)(d)\right) \notin s \\
& \text { or }\left(x, y \circ f_{i}\right) \in s^{\smile} \text { and }\left(x(c),\left(y \circ f_{i}\right)(d)\right) \notin s^{\smile} \text {, }
\end{aligned}
$$

that is,

$$
\begin{array}{rlll}
\text { either } & \left(\operatorname{id}_{M}, f_{i}\right) \in(x \times y)^{-1}(s) & \text { and } & \left(c, f_{i}(d)\right) \notin(x \times y)^{-1}(s) \\
\text { or } & \left(\operatorname{id}_{M}, f_{i}\right) \in(x \times y)^{-1}\left(s^{\smile}\right) & \text { and } & \left(c, f_{i}(d)\right) \notin(x \times y)^{-1}\left(s^{\smile}\right) .
\end{array}
$$

For every $i \in I_{a}$, recall that $\left(\mathrm{id}_{M}, f_{i}\right) \in r \wedge\left(c, f_{i}(d)\right) \notin r \Leftrightarrow \mathbf{r} \in S_{c d}^{i}$, for every $\mathbf{r} \leqslant \underline{\mathbf{M}}^{2}$. Thus, $s \in U$ if and only if there exist $x, y \in$ Aut $\underline{\mathbf{M}}$ and $i \in I_{a}$ such that either $(x \times y)^{-1}(\mathbf{s}) \in S_{c d}^{i}$ or $(x \times y)^{-1}\left(\mathbf{s}^{\smile}\right) \in S_{c d}^{i}$, if and only if there exist $x, y \in$ Aut $\underline{\mathbf{M}}$ and $\mathbf{r} \in \bigcup_{i \in I_{a}} S_{c d}^{i}$ such that either $s$ or $s^{\smile}$ is of the form $(x \times y)(r)$.

Finally we get the result below. 
PROPOSITION 4.8. Let $U$ be an aut-free globally minimal failset. Then

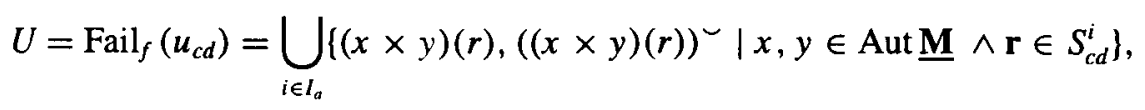

for some $f \in$ End $\underline{\mathbf{M}}$ where $c, d \in M$ satisfy $\{c, d\}=\left\{a, a^{-}\right\}$, for some a $\in \mathscr{J}_{k}$.

For every $a \in \mathscr{J}_{\kappa}$, let

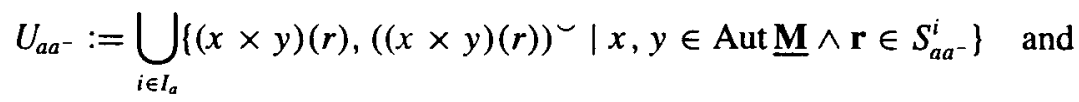

$$
\begin{aligned}
& U_{a^{-a}}:=\bigcup_{i \in I_{a}}\left\{(x \times y)(r),((x \times y)(r))^{\smile} \mid x, y \in \text { Aut } \underline{\mathbf{M}} \wedge \mathbf{r} \in S_{a^{-a} a}^{i}\right\} .
\end{aligned}
$$

Given a cover $\kappa \vee \theta\left(a, a^{-}\right)$of $\kappa$, with $a \in \mathscr{J}(\underline{\mathbf{M}})$, note that, for every $c \in \mathscr{J}(\underline{\mathbf{M}})$ such that $\kappa \vee \theta\left(a, a^{-}\right)=\kappa \vee \theta\left(c, c^{-}\right)$, we have that

$$
\begin{aligned}
\operatorname{ker} f_{i} \vee \theta\left(a, a^{-}\right) & =\operatorname{ker} f_{i} \vee \kappa \vee \theta\left(a, a^{-}\right)=\operatorname{ker} f_{i} \vee \kappa \vee \theta\left(c, c^{-}\right) \\
& =\operatorname{ker} f_{i} \vee \theta\left(c, c^{-}\right), \quad \forall i \in\{1, \ldots, n\},
\end{aligned}
$$

which implies that $I_{a}=I_{c}$, and either $\left[a, a^{-}\right]_{\kappa}=\left[c, c^{-}\right]_{\kappa}$ or $\left[a, a^{-}\right]_{\kappa}=\left[c^{-}, c\right]_{\kappa}$ (see [6, Lemma 1.7]). Thus we also have either $\left[a, a^{-}\right]_{f_{i}}=\left[c, c^{-}\right]_{f_{i}}$ for every $i \in\{1, \ldots, n\}$ or $\left[a, a^{-}\right]_{f_{i}}=\left[c^{-}, c\right]_{f_{i}}$ for every $i \in\{1, \ldots, n\}$. Consequently, either $S_{a a^{-}}^{i}=S_{c c^{-}}^{i}$ for every $i \in I_{a}$ or $S_{a a^{-}}^{i}=S_{c^{-c}}^{i}$ for every $i \in I_{a}$.

Finally, we may observe that, for each cover $\theta$ of $\kappa$ in $\operatorname{Con} \underline{\mathbf{M}}$, we have at most two candidates for aut-free globally minimal failsets that are $U_{a a^{-}}$and $U_{a^{-} a}$, where $a$ is any element of $\mathscr{J}_{\kappa}$ that satisfies $\theta=\kappa \vee \theta\left(a, a^{-}\right)$. For applications we refer to [8, Chapter 5, Example 3].

If $\underline{\mathbf{M}}$ generates a congruence distributive variety and each of its subalgebras is subdirectly irreducible, we get further. Denoting by $R_{\min }$ the set of all binary relations that are minimal elements of some pe-free globally minimal failset, we can prove that an aut-free globally minimal failset must not intersect $R_{\text {min }}$ (see [8, pages 107 and 108]). Moreover we get

PROPOSITION $4.9([8,4.2 .10])$. Let $U$ be a subset of $\Omega$. Then $U$ is an aut-free globally minimal failset if and only if $U$ is a minimal element of the set of all sets $U_{c d}$, where $c, d \in M$ satisfy the following conditions:

(a) there exists $a \in \mathscr{J}_{\kappa}$ such that $\{c, d\}=\left\{a, a^{-}\right\}$;

(b) $R_{\min }$ does not intersect $\bigcup_{i \in l_{a}}\left\{r \mid \mathbf{r} \in S_{c d}^{i}\right\}$.

In case $\operatorname{VSP}(\underline{\mathrm{M}})$ is a variety, the congruence $\kappa$ is the kernel of some endomorphism $f$ in End $\underline{\mathbf{M}} \backslash$ Aut $\underline{\mathbf{M}}$. Then, either $R_{\min }$ intersects $\bigcup_{i \in I_{a}}\left\{r \mid \mathbf{r} \in S_{a a^{-}}^{i}\right\}$ and 
$\bigcup_{i \in I_{a}}\left\{r \mid \mathbf{r} \in S_{a^{-} a}^{i}\right\}$, or at least one of the sets $U_{a a^{-}}$and $U_{a^{-} a}$ is an aut-free globally minimal failset, with $a \in \mathscr{J}_{\kappa}$. Thus, either there are no globally minimal failsets

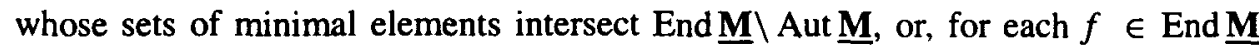
such that $\operatorname{ker} f=\kappa$, the set $\{f\}$ is a transversal of the set of aut-free globally minimal failsets. Hence $G \cup\{f\}$, where $G$ is a minimal generating set of Aut $\underline{\mathbf{M}}$, is a transversal of the set of all globally minimal failsets whose minimal elements are endomorphisms of $\underline{\mathbf{M}}$ or converses of their graphs.

\section{Acknowledgement}

The author wishes to thank Hilary Priestley for many instructive discussions and for her support and encouragement.

\section{References}

[1] D. M. Clark and B. A. Davey, Natural dualities for the working algebraist (Cambridge University Press, Cambridge, 1998).

[2] B. A. Davey, M. Haviar and H. A. Priestley, 'The syntax and semantics of entailment in duality theory', J. Symbolic Logic 60 (1995), 1087-1114.

[3] B. A. Davey and H. A. Priestley, 'Optimal natural dualities', Trans. Amer. Math. Soc. 338 (1993), $655-677$.

[4] _ , 'Optimal natural dualities II: general theory', Trans. Amer. Math. Soc. 348 (1996), 36733711.

[5] B. A. Davey and H. Werner, 'Dualities and equivalences for varieties of algebras', in: Contributions to lattice theory (Szeged, 1980) (eds. A. P. Huhn and E. T. Schmidt), Colloq. Math. Soc. János Bolyai 33 (North-Holland, Amsterdam, 1983) pp. 101-275.

[6] M. J. Saramago, 'A note on the covers of congruences of a finite lattice-structured algebra', preprint.

[7] — 'Optimal natural dualities for some quasivarieties of distributive double $p$-algebras', Acta Math. Univ. Comenian. (N.S.) 67 (1998), 249-271.

[8] - A study of natural dualities, including an analysis of the structure of failsets (Ph.D. Thesis, Universidade de Lisboa, Portugal, 1999).

[9] M. J. Saramago and H. A. Priestley, 'Optimal natural dualities; the structure of failsets', Internat. J. Algebra Comput. 12 (2002), 407-436.

[10] C. Wegener, Natural dualities for varieties generated by lattice-structured algebras (Ph.D. Thesis, University of Oxford, 1999).

Departamento de Matemática

Faculdade de Ciências

Universidade de Lisboa

1749-016 Lisboa

Centro de Álgebra Universidade de Lisboa

Portugal Av. Prof. Gama Pinto 2 1649-003 Lisboa

e-mail: matjoao@ptmat.fc.ul.pt 